\title{
Receptor-induced transient responses in cells with oscillatory actin dynamics
}

\author{
Jose Negrete, Jr. $\odot,{ }^{1,2,3}$ Alain Pumir $\odot,{ }^{3,4}$ Christian Westendorf $\odot,{ }^{3}$ Marco Tarantola, ${ }^{3}$ \\ Eberhard Bodenschatz, ${ }^{3,5,6}$ and Carsten Beta $\odot^{3,7, *}$ \\ ${ }^{1}$ Max Planck Institute for the Physics of Complex Systems (MPIPKS), D-01187 Dresden, Germany \\ ${ }^{2}$ École Polytechnique Fédérale de Lausanne (EPFL), CH-1015 Lausanne, Switzerland \\ ${ }^{3}$ Max Planck Institute for Dynamics and Self-Organization (MPIDS), D-37077 Göttingen, Germany \\ ${ }^{4}$ Laboratoire de Physique, Ecole Normale Supérieure de Lyon, Université de Lyon 1 and Centre National de la Recherche Scientifique, \\ F-69007 Lyon, France \\ ${ }^{5}$ Institute for Dynamics of Complex Systems, University of Göttingen, D-37073 Göttingen, Germany \\ ${ }^{6}$ Laboratory of Atomic and Solid-State Physics and Sibley School of Mechanical and Aerospace Engineering, \\ Cornell University, Ithaca, New York 14853, USA \\ ${ }^{7}$ Institute of Physics and Astronomy, University of Potsdam, D-14476 Potsdam, Germany
}

(Received 12 September 2019; accepted 22 January 2020; published 2 March 2020)

\begin{abstract}
Living cells adjust their sensing and migratory machinery in response to changes in their environment. In this work, we show that cells of the social amoeba Dictyostelium discoideum modulate the dynamical state of their actin cytoskeleton in response to an external pulse of the chemoattractant cyclic adenosine monophosphate (cAMP). In particular, we focus on a population of cells that exhibits noisy oscillatory cycles of actin polymerization and systematically study receptor-induced transitions in their cytoskeletal dynamics. In response to a short external pulse of cAMP, these cells adopt a noisy quiescent state, before returning to their initial, oscillatory dynamics. The response exhibits a biphasic time profile, with a duration that shows strong variability between cells; it can extend as long as approximately twelve oscillation cycles. We propose a model that is based on a generic nonlinear noisy oscillator. Our theoretical analysis suggests that the transient termination of oscillations in response to a receptor stimulus occurs via a Hopf bifurcation.
\end{abstract}

DOI: 10.1103/PhysRevResearch.2.013239

\section{INTRODUCTION}

Amoeboid cells constantly migrate and probe the chemical composition of their surroundings, in order to respond to changing environmental conditions. For instance, individual cells of the social amoeba Dictyostelium discoideum (D. discoideum) react to extracellular cAMP, emitted by neighboring D. discoideum cells, to aggregate and self-organize into a multicellular fruiting body. The extracellular cAMP is detected by the corresponding $\mathrm{G}$ protein coupled receptors that trigger a cascade of intracellular signaling events, involving Ras activation and the phosphorylation of PIP2 to PIP3. This induces reorganization of the actin cytoskeleton and chemotactic migration of cells towards regions of higher cAMP concentration [1]. The cytoskeletal actomyosin system not only generates the forces required for amoeboid locomotion but also determines the shape and mechanical stability of the cells $[2,3]$.

\footnotetext{
*beta@uni-potsdam.de

Published by the American Physical Society under the terms of the Creative Commons Attribution 4.0 International license. Further distribution of this work must maintain attribution to the author(s) and the published article's title, journal citation, and DOI. Open access publication funded by the Max Planck Society.
}

In our previous work, we have probed the dynamical properties of actin polymerization in response to external pulses of cAMP [4]. We found that the polymerizationdepolymerization dynamics at the cell cortex is reminiscent of a damped oscillator and the response to a cAMP pulse saturates above a critical cAMP level [5]. A subpopulation of the investigated cells exhibits self-sustained oscillations in the polymerization-depolymerization dynamics, leading to the conjecture that the actin cytoskeleton operates close to an oscillatory instability $[4,6]$. Being close to criticality is a global property shared by many other cellular systems and in different biological contexts [7-9]. Besides, self-sustained oscillations are an example of the rich autonomous dynamics of the cytoskeleton and its governing chemotactic signaling system [10,11]. Other examples include intracellular traveling waves and spontaneous pseudopod formation resulting in random motility and reorientation [12]. These autonomous processes can be directly influenced by receptor-mediated chemoattractant stimuli [13]. In this work, we focus on the subpopulation of cells which show self-sustained oscillations (see Fig. 1 for an example) and probe their response to a chemoattractant stimulus. Based on a combination of experiments and modeling, we characterize the dynamical transition induced by a change in the ambient cAMP concentration. We report that a single short pulse of $\operatorname{cAMP}(\approx 1 \mathrm{~s})$ induces a transition of the actin cytoskeleton in $D$. discoideum from self-sustained oscillations to a nonoscillatory state. After a 

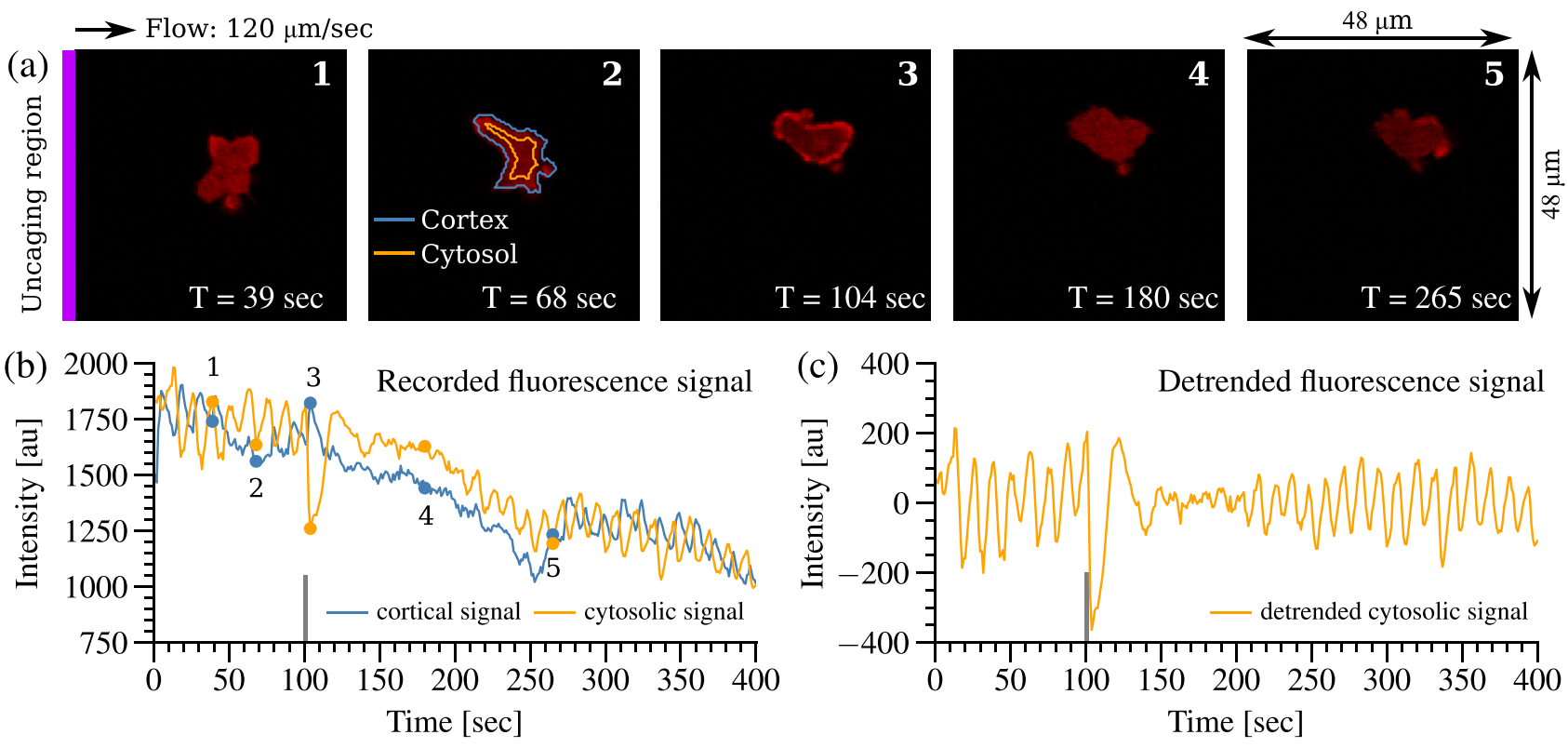

FIG. 1. Experimental set-up and data analysis. (a) Exemplary image series. The $D$. discoideum cell is centered within a $48 \times 48 \mu \mathrm{m}$ imaging region. The cAMP stimulus is released upstream of the cell by a line scan of a 405-nm laser (uncaging region, purple) and advected by the flow toward the cell (mean velocity $=120 \mu \mathrm{m} / \mathrm{s}$ ). The imaged LimE-mRFP fluorescence is segmented into cortical and cytosolic signals (displayed in frame 2, blue and yellow, respectively). (b) Averaged fluorescence intensity of the cortical (blue) and the cytosolic region (yellow) over time. The respective frames in panel (a) are marked by blue and yellow dots. (c) Cytosolic signal after detrending. The cAMP stimulus was applied at $t=100 \mathrm{~s}$ and is denoted by a gray bar in panels (b) and (c).

transient time of varying length, the actin cytoskeleton returns back to its initial oscillatory state, showing that the $D$. discoideum amoeba may change their dynamical state as a response to an external chemoattractant stimulus.

We use the flow photolysis method to stimulate single cells with short-time pulses of cAMP. This method relies on the photoactivation of caged compounds in microfluidic channels $[14,15]$. The cytoskeletal actin response is recorded as changes in the subcellular LimE-mRFP distribution [4]. This Lim-domain protein is a marker for filamentous actin $[16,17]$ and reflects changes in cortical actin polymerization by translocating between the cytosol and the cell cortex [18]; see Fig. 1(a). The transient response of a $D$. discoideum amoeba to a sudden change in ambient cAMP concentration can be divided into two phases (biphasic response). First, both the signaling system and the actin cytoskeleton are uniformly activated along the cell membrane with a characteristic duration of approximately $20 \mathrm{~s}$ [19-21]. This is followed by a second more irregular phase, during which localized patches of activity appear and disappear at random locations around the cell perimeter [22-25]. This response pattern is also observed for the oscillatory cells investigated here. Interestingly, during the second phase, which varies in duration between $\approx 40$ and $\approx 200 \mathrm{~s}$, the oscillatory dynamics stop before the cells recover their initial oscillatory state [see Fig. 1(c)].

The aim of this work is to characterize the dynamical transition behind this observation. Based on our previous findings that the actin cytoskeleton in $D$. discoideum operates close to a bifurcation point [4,6], we rationalize our results in the framework of a reductionist model. Here we rely on the well-known fact that close to a bifurcation point, the behavior of a dynamical system displays universal behavior, which is captured by the normal form of the corresponding transition $[26,27]$. For our present analysis, we use the normal form of a supercritical Hopf bifurcation, the so-called Stuart-Landau oscillator, as a starting point to describe actin oscillations close to the onset of periodic dynamics and subject to additive noise. In addition to the frequency of the underlying oscillations, the model introduces three parameters: $\lambda$ characterizes the distance to the bifurcation (i.e., the transition point to the nonoscillatory state), $D$ gives the amplitude of the noise, and $g$ sets the strength of the nonlinearity. Based on the dimensionless ratio $\lambda / \sqrt{g D}$, we can quantify the relative impact of deterministic oscillations and noise on the overall dynamics. This model provides a good description of oscillatory cells in the absence of any stimulation [6] and is now extended to account for the cAMP-induced dynamical transition to a nonoscillatory state.

\section{EXPERIMENTAL AND ANALYSIS METHODS}

\section{A. Experimental setup and protocol}

D. discoideum cell culture and preparation for an experiment were carried out as previously described [4,6]. The starved cells were injected into the microfluidic channel $(500 \mu \mathrm{m} \times 30 \mathrm{~mm} \times 26 \mu \mathrm{m})$ and allowed to settle for 15 min. Afterward, the phosphate buffer (PB) was replaced with a solution of $10 \mu \mathrm{M}$ BCMCM-caged cAMP in PB (Biolog, Bremen, Germany). A 250- $\mu$ l syringe (Hamilton, Bonaduz, Switzerland), mounted on a standard infuse syringe pump (Harvard Apparatus PHD 2000, Holliston, MA, USA) was used to insert the caged compound at a constant flow rate of $5 \mu \mathrm{l} / \mathrm{h}$, corresponding to an average flow velocity of approximately $120 \mu \mathrm{m} / \mathrm{s}$. 

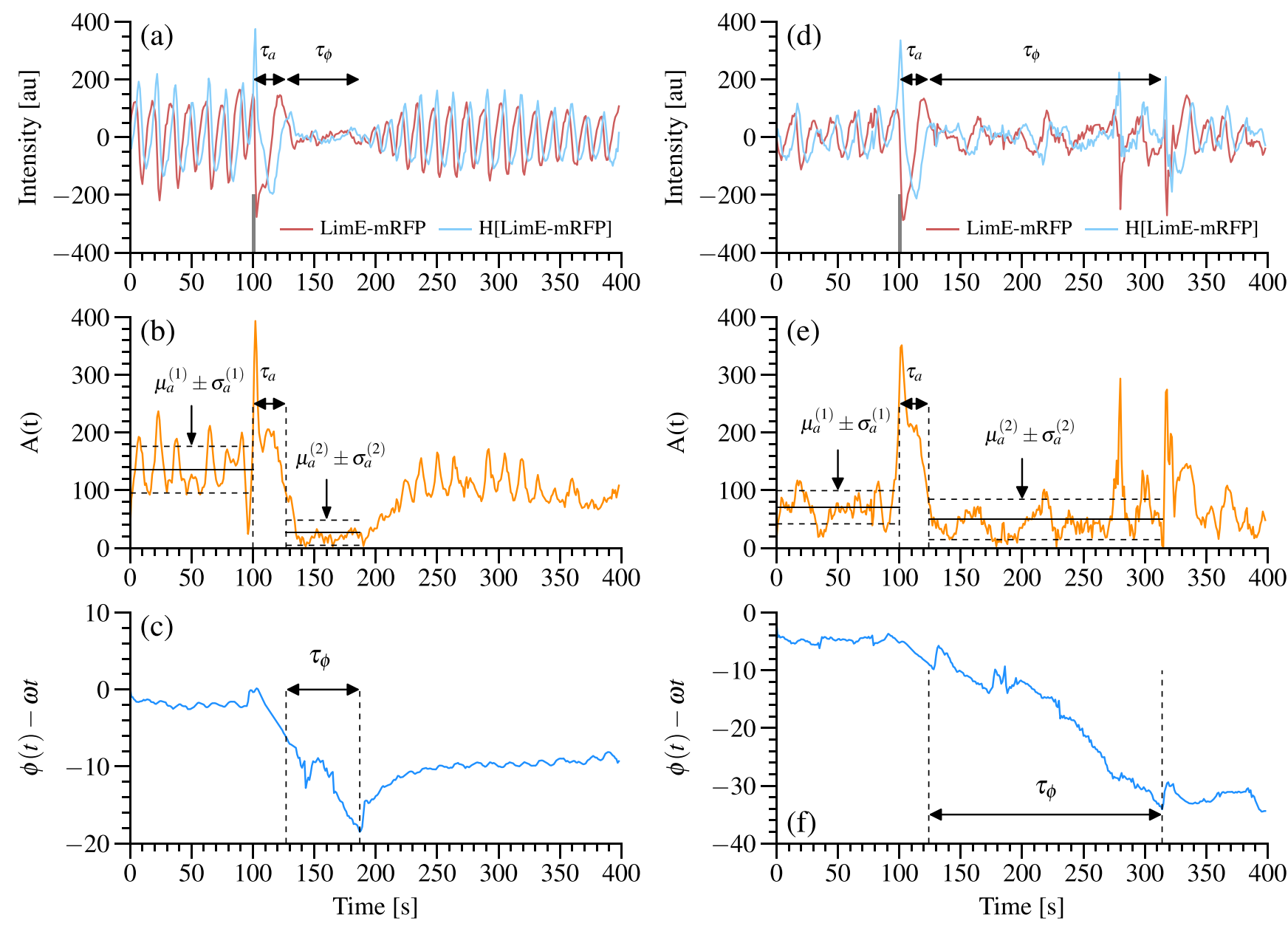

FIG. 2. Examples of single cell $D$. discoideum LimE-mRFP responses to a short pulse of cAMP. The two columns correspond to two different cells. Panels (a) and (d) show the detrended fluorescence time traces of LimE-mRFP (red) and their corresponding Hilbert transforms (blue). The cells are stimulated at $\tau_{\text {stim }}=100 \mathrm{~s}$ with a pulse of cAMP for one second (the stimulation time is denoted by a gray bar). The evolution of the amplitudes of the time traces are shown in panels (b) and (e), and the corresponding phase drifts are given in panels (c) and (f). The response to cAMP is divided into two phases. The duration of the first phase, the amplitude transient time $\tau_{a}$, is defined by the time it takes to return to $25 \%$ of the response amplitude maximum. The duration of the second phase, which we call the phase transient time $\tau_{\phi}$, is defined as the time interval between the end of the first phase and recovery of the phase $\phi(t)$ back to its main frequency [shown in panels (c) and (f)]. The amplitude mean (black line) and standard deviation (dashed lines) are calculated over the first $100 \mathrm{~s}$ and in the second phase of the transient response, shown in panels (b) and (e).

In this work, we analyzed fluorescence images of LimEmRFP [28], which labels polymerized actin, in combination with either Coronin-GFP [29] or Aip1-GFP [30]. The fluorophores were imaged with an Olympus FV-1000 inverted confocal laser scanning microscope, using an UPLSAPO $60 \mathrm{X}$ oil immersion objective. The mRFP was excited by the 543-nm line of a multiline 1-mW HeNe laser (Melles Griot, Carlsbad, CA, USA). Each cell was imaged individually in a region of $48 \times 48 \mu \mathrm{m}[120 \times 120$ pixels, Fig. 1(a)], with the confocal plane set approximately $2 \mu \mathrm{m}$ above the cover slip surface. At the upstream side of the imaging region, a line of $0.4 \times 40 \mu \mathrm{m}$ [1 $\times 120$ pixels, Fig. 1(a), panel 1, pink] was scanned by a $405-\mathrm{nm}$ laser diode to photochemically release the cAMP from its caged precursor (see flow photolysis, described in Refs. [14,31]). The cAMP was transported by the fluid flow toward the cell, which was centered at a distance of about $20 \mu \mathrm{m}$ downstream of the uncaging region.
During each experiment, the cell was first imaged for $100 \mathrm{~s}$ in the absence of any cAMP stimulation to record the initial activity of the actin cytoskeleton (imaging frame rate: $1 \mathrm{~Hz}$ ). These $100 \mathrm{~s}$ of each data set were previously presented and analyzed in Ref. [6]. At $\tau_{\text {stim }}=100 \mathrm{~s}$, we initiated the uncaging laser to stimulate the cell with an external pulse of cAMP for $1 \mathrm{~s}$ and continued the recordings for a time interval of 100 to $300 \mathrm{~s}$.

\section{B. Data analysis}

The LimE-mRFP images were segmented into two regions, indicated in Fig. 1(a, panel 2). The cortex is defined as a $1.2-\mu \mathrm{m}$-thick region at the cell's outer edge (blue), while the cytosol is defined as the remainder of the cell's interior (yellow). The mean of each region yields the average fluorescence that is shown over time in Fig. 1(b). Each cytosolic time trace was detrended by subtracting a moving average 
[Fig. 1(c), window size of $40 \mathrm{~s}$ ]. The data sets were divided into oscillatory and nonoscillatory time traces by calculating the autocorrelation function of the first $100 \mathrm{~s}$ prior to cAMP stimulation. Only time traces with an autocorrelation function that showed at least one oscillation cycle with a period below 33.33 s (i.e., $1 / 3$ of the time window before $\tau_{\text {stim }}$ ) were categorized as oscillatory and selected for further analysis. We furthermore required that the largest peak that occurs within the first $33.33 \mathrm{~s}$ of the autocorrelation reaches at least $10 \%$ of the initial value of the autocorrelation function. In addition, we excluded cells that did not show any response to the cAMP stimulus in the amplitude or phase signal.

Out of a total of 165 recorded cells, 26 passed this stringent selection protocol $(\approx 15 \%)$ and were included in the subsequent analysis. The corresponding time traces and their respective autocorrelation functions are shown in Figs. 11-14 in the Appendix. We also computed the Hilbert transform of the LimE-mRFP time trace for each case; an example is shown in blue in Figs. 2(a) and 2(d). This allowed us to calculate the amplitude $A(t)$ [Figs. 2(b) and 2(e)] and phase $\phi(t)$ [Figs. 2(c) and 2(f)] as a function of time. The majority of cases (22 cells) show a deceleration of the phase after stimulus. Only four cases, displayed in the four last rows of Fig. 14 in the Appendix, exhibit an accelerating phase after stimulation.

Based on the time traces of amplitude and phase records, we can then define the timescales of the two phases of the response (biphasic response profile). The duration of the first phase is defined as the interval between the time point of stimulation and the time point when the amplitude has dropped back to $25 \%$ of its maximal value. We call this timescale the amplitude transient time $\tau_{a}$. The time evolution of the phase $\phi(t)$ is well captured by

$$
\phi(t)=\omega_{o} t+\phi_{o}+\eta(t),
$$

where $\omega_{o}$ is the central frequency of the time series, $\phi_{o}$ is the initial phase value, and $\eta(t)$ is the deviation from the linear evolution caused by noise. We fitted the first $100 \mathrm{~s}$ of the phase with a simple functional form $\omega t+\phi_{o}^{\prime}$, where $\omega$ and $\phi_{o}^{\prime}$ are determined by minimizing the difference between the signal $\phi(t)$ and the linear dependence $\omega t+\phi_{o}^{\prime}$ ( $L_{2}$ norm of $\left.\phi-\omega t-\phi_{o}^{\prime}\right)$. Elementary algebra shows that

$$
\omega=\frac{12}{t_{\mathrm{obs}}^{2}}\left[\overline{\phi(t) t}-\frac{\overline{\phi(t)} t_{\mathrm{obs}}}{2}\right],
$$

where we denote $\omega$ as the detected frequency and the overbar as the average over the observation time $t_{\text {obs }}$. The detected frequency $\omega$ might differ from the value of the central frequency $\omega_{o}$, and the error between them will depend on $t_{\mathrm{obs}}$.

To determine the duration of the second phase of the cellular cAMP response, we analyzed the phase $\operatorname{drift} \phi(t)-\omega t$. This quantity drifts almost linearly during the transient phase, followed by a recovery toward a constant value. The time interval between the end of $\tau_{a}$ and the end of the phase drift is defined as the phase transient time $\tau_{\phi}$. Note that in 9 out of the total number of 26 cases, the phase transient time $\tau_{\phi}$ exceeds the duration of the recording and cannot be determined. For this reason, the analysis of the phase transient time $\tau_{\phi}$ is based on 17 records only, whereas 26 data points are available in all other cases.

\section{MODELING THE ACTIN CYTOSKELETON DYNAMICS: FAST OSCILLATIONS AND SLOW RESPONSES}

In this section, we present an extension of the model introduced in Ref. [6], to capture the transient response of oscillatory cells to a pulse of cAMP that was described above. A detailed comparison between the model and the experimental data will be provided in Sec. IV. We briefly recall the structure of the original model [6]. Namely, the system is described by a complex variable, $z=x+i y$, satisfying the equation

$$
\frac{d z}{d t}=\left[\lambda+i \omega_{o}\right] z-g|z|^{2} z+\xi(t) .
$$

The real part $\operatorname{Re}[z]=x$ corresponds to the mean fluorescence emitted by polymerization markers at time $t$. The imaginary part, $\operatorname{Im}[z]=y$, can be viewed as a negative feedback acting on them. The parameter $\omega_{0}$ is the central frequency of the oscillator. In the absence of noise $(\xi=0)$, the resting state, $z=0$, is a solution, whose stability is given by the sign of $\lambda$. For $\lambda>0$, the resting state is unstable with respect to small perturbations, and the system displays a stable limit cycle. The amplitude of the limit cycle oscillations is set by a balance between the amplification rate and the nonlinearity, $g|z|^{2} z$, and scales as $\sim \sqrt{\lambda / g}$. For $\lambda<0$ the resting state is stable and oscillations are damped. The noise, $\xi(t)=\xi_{R}(t)+i \xi_{I}(t)$, is taken to be Gaussian, with short time correlations that are white in time in the present analysis,

$$
\left\langle\xi_{i}(t) \xi_{j}\left(t^{\prime}\right)\right\rangle=2 D \delta\left(t-t^{\prime}\right) \delta_{i j},
$$

where $i, j$ refer to the indices $R$ and $I$. The response in actin polymerization to an external pulse of cAMP is captured by introducing two additional components that exert an incoherent feed forward type regulation [32]. The first one corresponds to the receptor-induced response of a secondary messenger $s(t)$ that indirectly activates actin polymerization. The second component represents a polymerization inhibitor $w(t)$ that is activated by $s(t)$. The corresponding interaction network is summarized in Fig. 3(a). The model given by Eq. (3) is then extended as

$$
\frac{d z}{d t}=\left[\tilde{\lambda}(w(t))+i \omega_{o}\right] z-g|z|^{2} z-\frac{s(t)}{\tau_{r}}+\xi(t) .
$$

The stimulus, $s(t)$, has the following functional form,

$$
s(t)=s_{o}\left(\frac{t-\tau_{\text {stim }}}{\tau_{s}}\right)^{4} e^{-\frac{t-\tau_{\text {stim }}}{\tau_{s}}} \Theta\left(t-\tau_{\text {stim }}\right),
$$

where $\Theta(t)$ corresponds to the Heaviside function $(\Theta(t)=$ 0 for $t<0$, and $\Theta(t)=1$ for $t>0)$. Its time evolution is shown in the inset of Fig. 3(b), which corresponds to the response profile of the secondary messenger Ras, measured in Ref. [33]; see the Appendix for details. The stimulus acts directly on the oscillatory variable $x$ through the additive term $-s(t) / \tau_{r}$; this interaction is represented by a vertical arrow in Fig. 3(a). In addition, activation of the inhibitory component $w(t)$ proceeds with a characteristic response time $\tau$ according to

$$
\frac{d w}{d t}=\frac{1}{\tau}[s(t)-w] .
$$



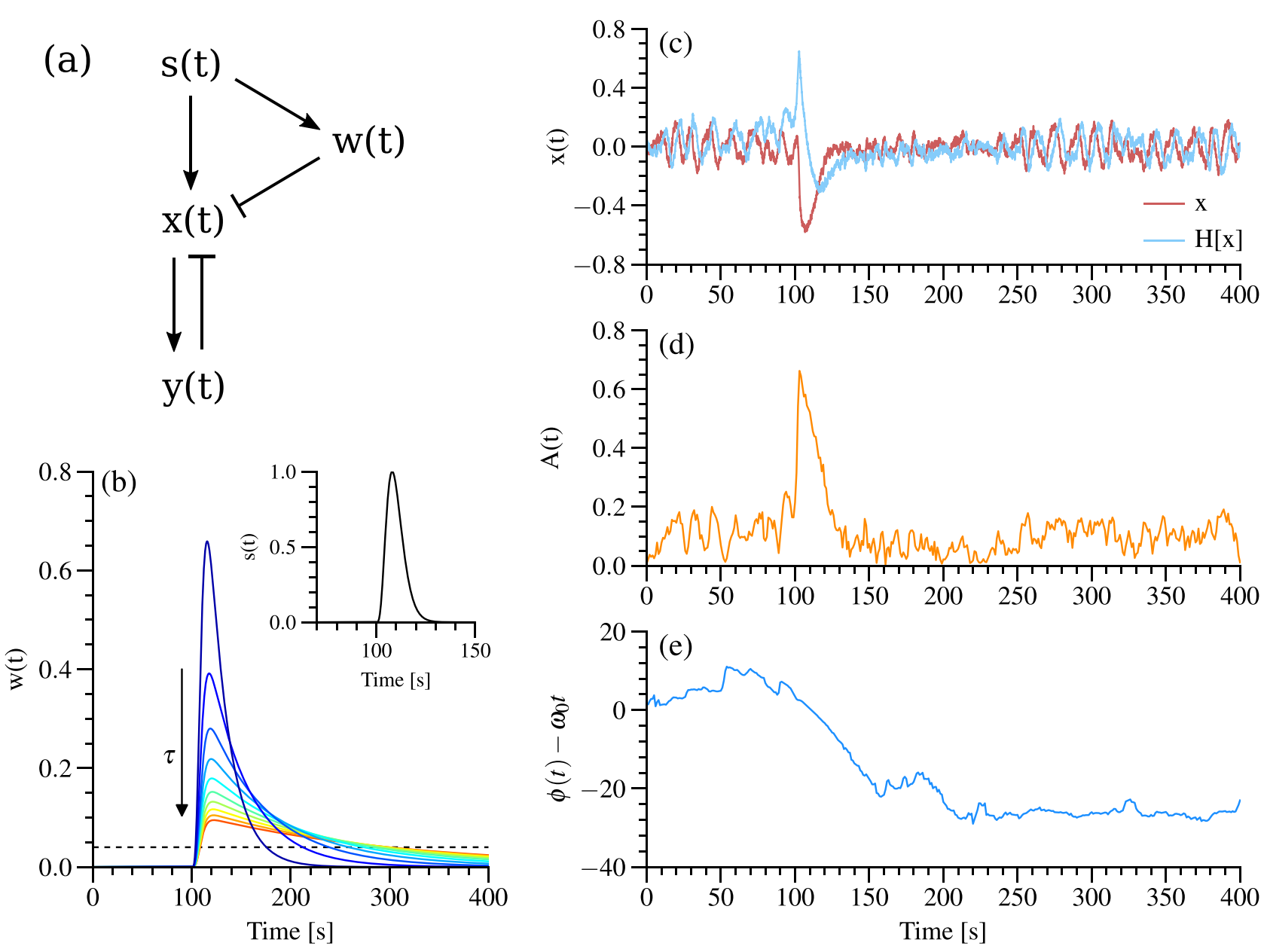

FIG. 3. Model for the response of the $D$. discoideum actin cytoskeleton to short time cAMP pulses. (a) Network topology of the proposed model. The signal $s(t)$ is activated by an external cAMP stimulation. The variable $x(t)$ describes the dynamics of LimE-mRFP (i.e., the actin polymerization) and $y(t)$ is the negative feedback, leading to spontaneous oscillations in the absence of an external stimulus. The variable $w(t)$ acts as an inhibitor which determines the duration of the transient response. (b) The response of $w(t)$ elicited by the signal $s(t)$ (inset). Several realizations with different values of $\tau(25 \mathrm{~s} \leqslant \tau \leqslant 200 \mathrm{~s})$ are shown. (c) A single numerical realization of the dynamics of $x(t)$ (red) and its Hilbert transform (blue) with the corresponding amplitude $A(t)$ (d) and phase drift $\phi-\omega_{o} t(\mathrm{e})$.

The inhibitory action of $w(t)$ on the dynamics of $z$ occurs via a modulation of $\tilde{\lambda}(w(t))$ given by

$$
\tilde{\lambda}(w(t))=\lambda-\lambda_{\rho} \Theta(w(t)-K) .
$$

Effectively, the variable $\lambda$ is reduced by a positive quantity $\lambda_{o}$ as long as $w(t)>K$, and returns to the original value when $w(t)<K$. Therefore, in this model, the inhibitory variable $w(t)$ controls the duration of the transient response. Figure 3(b) shows the response of $w(t)$ for different values of the relaxation time $\tau$. As $\tau$ increases, $w(t)$ becomes less sensitive to $s(t)$ and takes longer to relax back to its initial state.

As an example, Fig. 3(c) shows a single numerical realization of the oscillatory actin signal $x(t)$ that receives a stimulus at $t=100 \mathrm{~s}$, showing that the system transiently oscillates with a reduced amplitude after stimulation. The corresponding amplitude $A(t)$ and phase drift $\phi(t)-\omega_{o} t$ are shown in Figs. 3(d) and 3(e), respectively. The behavior of the numerical solution is qualitatively similar to our experimental results; see Fig. 2. In the Appendix, we show several examples of how individual experimental time traces can be associated with numerical realizations of the model, demonstrating that the model is able to reproduce details of the experimental data; see Fig. 15 in the Appendix.

In the model, the relaxation time $\tau$ of the inhibitory variable $w(t)$ determines the total duration of the biphasic response $T=\tau_{a}+\tau_{\phi}$. The dependence of $T$ on the relaxation time $\tau$ in the model is shown in Fig. 7(b), given a fixed value of $K$. To model the variability in the duration $T$ of the biphasic response observed in experiments, we assume, for simplicity, that the relaxation time $\tau$ is distributed exponentially,

$$
\rho(\tau)=\frac{\Theta\left(\tau-\tau_{1}\right)}{\tau_{2}} e^{-\left(\tau-\tau_{1}\right) / \tau_{2}},
$$

with two fitting parameters $\tau_{1}$ and $\tau_{2}$. However, from the experiments the distribution $\rho(\tau)$ is not directly accessible. Only the distribution $\rho(T)$ of the total duration $T$ is known; see Fig. 7(c). We fitted the experimental distribution $\rho(T)$ by tuning the fitting parameters $\tau_{1,2}$ in the distribution $\rho(\tau)$, which determines the corresponding distribution $\rho(T)$ via 

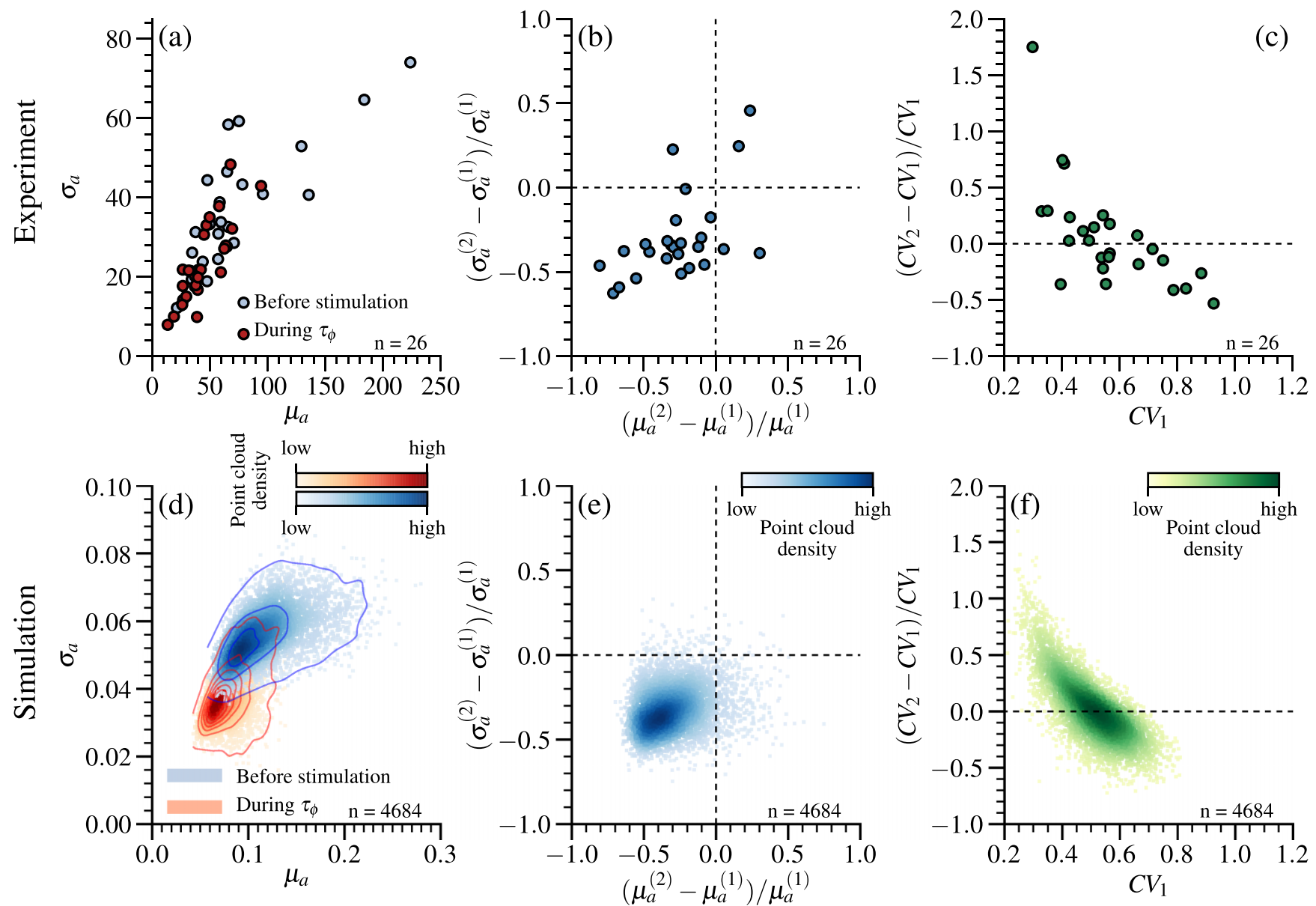

FIG. 4. Mean, standard deviation, and coefficient of variation in the amplitude fluctuations. Experimental data: (a) Mean amplitude $\mu_{a}$ and standard deviation $\sigma_{a}$ measured before stimulation (blue) and during $\tau_{\phi}$ (red). (b) Relative change of the mean amplitude vs relative change of the standard deviation. Superscript indices (1) and (2) denote the mean and standard deviation before stimulation and during $\tau_{\phi}$, respectively. (c) Changes in the amplitude coefficient of variation before stimulation $\left(\mathrm{CV}_{1}=\sigma_{a}^{(1)} / \mu_{a}^{(1)}\right)$ and during $\tau_{\phi}\left(\mathrm{CV}_{2}=\sigma_{a}^{(2)} / \mu_{a}^{(2)}\right)$. Model predictions: (d) Mean amplitude $\mu_{a}$ and standard deviation $\sigma_{a}$ derived from numerically evaluated time traces. Blue and red dots correspond to the time intervals before stimulation and during $\tau_{\phi}$, respectively. (e) Relative change of the mean amplitude vs relative change of the standard deviation. (f) Changes in the amplitude coefficient of variation before stimulation $\left(\mathrm{CV}_{1}\right)$ and during $\tau_{\phi}\left(\mathrm{CV}_{2}\right)$. For all point clouds in panels (d), (e), and (f), a kernel density estimate of the distribution was calculated and the color gradient indicates the density of points. Additionally, contours at different density levels were drawn into panel (d), as both point clouds are partially overlapping.

relation (A1); see also the Appendix for a detailed presentation of the fitting procedure. In our numerical simulations, we then draw for each simulation a different value of $\tau$ from the distribution $\rho(\tau)$.

Previously, we have shown that the oscillatory actin dynamics follows Eq. (3), where each cell is characterized by a different value of the dimensionless parameter $\Lambda=$ $\lambda / \sqrt{g D}$ [6]. Therefore, in simulations we assigned to each cell a different value of $\Lambda$, drawn from the following distribution,

$$
\rho(\Lambda)=\frac{1}{\sqrt{2 \pi \sigma_{\Lambda}^{2}}} e^{-\Lambda^{2} / 2 \sigma_{\Lambda}^{2}},
$$

where $\sigma_{\Lambda}^{2}=2.45$; see Fig. 7(d). All other parameters in Eqs. (5)-(8) are set to constant values that are given in the Appendix.

\section{EXPERIMENTS AND MODELING RESULTS}

\section{A. Statistics of transient times and amplitude analysis}

We first focus on the statistics of the amplitude and phase transient times. From the experiments, we find for the amplitude transient times a mean value and standard deviation of $\left\langle\tau_{a}\right\rangle=28.6 \mathrm{~s}$ and $\sigma_{\tau_{a}}=14.03 \mathrm{~s}$, respectively, yielding a coefficient of variation of $\sigma_{\tau_{a}} /\left\langle\tau_{a}\right\rangle=0.49$. The phase transient times $\left(\tau_{\phi}\right)$ show a larger spreading $\left(\left\langle\tau_{\phi}\right\rangle \pm \sigma_{\tau_{\phi}}=\right.$ $65.1 \pm 48.79 \mathrm{~s})$ with a coefficient of variation of $\sigma_{\tau_{\phi}} /\left\langle\tau_{\phi}\right\rangle=$ 0.74 . The largest phase transient time observed is more than two times larger than the largest amplitude transient time $\left(\max \left[\tau_{\phi}\right]=2.11 \max \left[\tau_{a}\right]\right)$. From our numerical simulations, we obtained $\left\langle\tau_{a}\right\rangle \pm \sigma_{\tau_{a}}=28.8 \pm 1.91 \mathrm{~s}$ and $\left\langle\tau_{\phi}\right\rangle \pm \sigma_{\tau_{\phi}}=$ $57.7 \pm 32.64 \mathrm{~s}$ for the mean values and standard deviations of the amplitude and phase transient times, which agree well with our experimental findings (see also the Appendix for further details). 

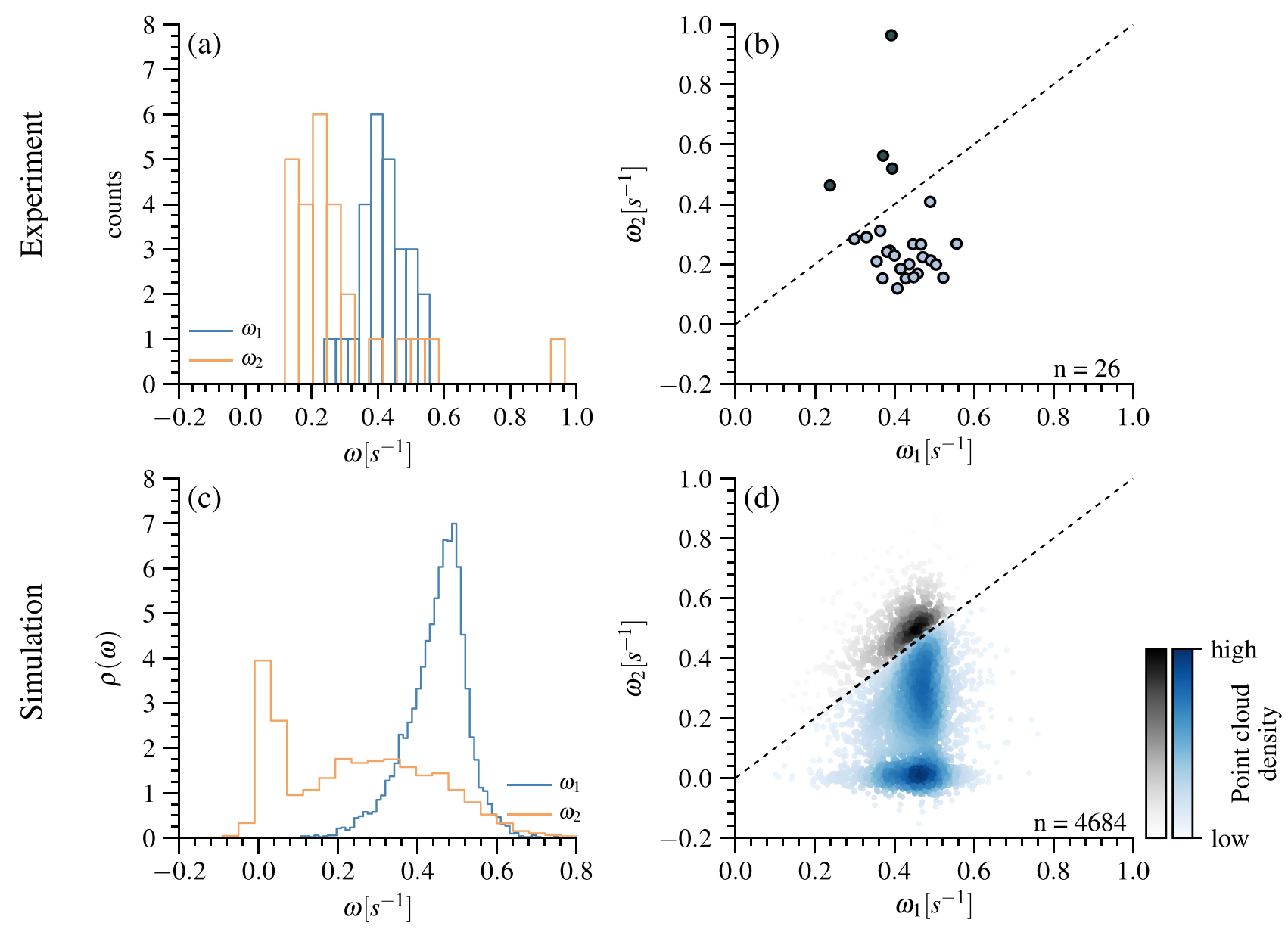

FIG. 5. Distributions of the detected frequencies. (a) Histograms of $\omega_{1}$ (before stimulation) and $\omega_{2}$ (during $\tau_{\phi}$ ) obtained from the experimental data and (b) scatter plot of $\omega_{1}$ vs $\omega_{2}$. The dashed line corresponds to $\omega_{2}=\omega_{1}$. (c) Histograms of $\omega_{1}$ and $\omega_{2}$ resulting from the numerical simulations. (d) Scatter plot of $\omega_{1}$ vs $\omega_{2}$ for the numerical data. The color gradient indicates the density of the point cloud, calculated by a kernel density estimate of the distribution. Cases in panels (b) and (d) for which $\omega_{1}>\omega_{2}\left(\omega_{1}<\omega_{2}\right)$ are shown in blue (black).

We furthermore determined the mean and standard deviation of the amplitude of the detrended LimE-mRFP fluorescence signal prior to stimulation $\left(\mu_{a}^{(1)}\right.$ and $\left.\sigma_{a}^{(1)}\right)$ and during the second phase of the cAMP response $\left(\mu_{a}^{(2)}\right.$ and $\sigma_{a}^{(2)}$ ) from the experimental data; see Figs. 2(b) and 2(e). Figure 4(a) shows the standard deviation as a function of the amplitude mean before stimulation (blue) and during the second phase of the response (red). The data indicate that the standard deviation $\sigma_{a}$ increases with the mean $\mu_{a}$. A direct comparison with the results of our model simulations can be seen in Fig. 4(d). The distribution after stimulus (red points) is more compact and centered at lower values compared to the distribution before stimulation (blue points) but overall we find good agreement with the experimental results. The distribution of $\sigma_{a}$ as a function of the mean $\mu_{a}$ before stimulus [Fig. 4(d), blue points] can be attributed to cell-to-cell variability. In the simulations, this is taken into account by including parameter distributions, such as, for example, the distribution of $\lambda / \sqrt{g D}$. We obtain a Pearson correlation coefficient between $\mu_{a}$ and $\sigma_{a}$ of 0.82 ( $p$ value $10^{-13}$ ) in the experiment and 0.70 ( $p$ value 0 ) for the numerical simulations.

In the majority of recorded cell responses, the mean and standard deviation of the F-actin fluorescence signal is decreased after stimulation. This can be seen from the rela- tive changes in mean and standard deviation, displayed in Fig. 4(b), where most data points lie in the lower left quadrant. Correlations between the duration of the responses and the corresponding amplitudes were not observed (see Fig. 10 in the Appendix). Also in this case, the simulations closely match the experimental results; see Fig. 4(e).

Finally, we quantified the coefficient of variation $\left(\mathrm{CV}_{i}=\right.$ $\left.\sigma_{a}^{(i)} / \mu_{a}^{(i)}\right)$, which measures the level of amplitude noise, where $i=1,2$ denotes the coefficient of variation before stimulation and during the second phase of the response. Changes in $\mathrm{CV}_{i}$ as measured in the experiment are displayed in Fig. 4(c) as a function of the coefficient of variation before stimulus, $\mathrm{CV}_{1}$. Roughly, half of the cells exhibited a noisier regime after stimulation, $\left(\mathrm{CV}_{2}-\mathrm{CV}_{1}\right) / \mathrm{CV}_{1}>0$, while the amplitude noise is reduced for the remaining cells. A qualitatively similar distribution is observed in the model simulations; see Fig. 4(f).

\section{B. Frequency analysis}

We now investigate the frequencies involved in the response to the chemottractant pulse. We denote the frequency detected before stimulation as $\omega_{1}$ and the frequency detected during the second phase of the response as $\omega_{2}$. The experimentally derived distributions of $\omega_{1}$ and $\omega_{2}$ are shown in Fig. 5(a) in blue and orange, respectively. The histogram of 
$\omega_{1}$ is peaked at $\omega_{o} \sim 2 \pi / 13 \mathrm{~s}^{-1}$ with a mean value of $\left\langle\omega_{1}\right\rangle=$ $2 \pi / 15.1 \mathrm{~s}^{-1}$ and a standard deviation of $\sigma_{\omega_{1}}=0.07 \mathrm{~s}^{-1}$, while the histogram of $\omega_{2}$ is irregularly shaped $\left(\left\langle\omega_{2}\right\rangle \pm \sigma_{\omega_{2}}=\right.$ $2 \pi / 28.0 \pm 0.06 \mathrm{~s}^{-1}$ ). Additionally, in Fig. 5(b) each cell is represented as a point in the $\left(\omega_{1}, \omega_{2}\right)$ plane to show that for the majority of cells the frequency of cortical oscillations decreases after stimulation.

In the numerical simulations of the model, we set the frequency $\omega_{o}$ in Eq. (5) to $\omega_{o}=2 \pi / 13 \mathrm{~s}^{-1}$. Before stimulation, the frequency $\omega_{1}$ determined from numerical simulations of the model fluctuates, with a mean value of $\left\langle\omega_{1}\right\rangle \pm \sigma_{\omega_{1}}=$ $2 \pi / 14.0 \pm 0.07 \mathrm{~s}^{-1}$; see Fig. 5(c). The good agreement between the distribution of frequencies in the experiment and in the model suggests that the dispersion in the recorded frequency is due to phase noise, as discussed in the Appendix.

After stimulation, the frequency $\omega_{2}$ predicted by the model is also fluctuating $\left(\left\langle\omega_{2}\right\rangle \pm \sigma_{\omega_{2}}=2 \pi / 35.4 \pm 0.15 \mathrm{~s}^{-1}\right)$; see Fig. 5(c). We note that for a noisy oscillator [Eq. (1)] with a small value of $\lambda / \sqrt{D g}$ as it occurs in the noise-dominated or weakly oscillatory case, the amplitude of $z$ can occasionally become zero, resulting in a phase jump (phase slip; see the Appendix for details). This makes the definition of the phase ambiguous, so that the observation of $\omega_{2}$ should be interpreted as an effective frequency. When $\left(\lambda-\lambda_{o}\right)$ is large and negative, the solution of the model is $z(t) \approx-\xi(t) /\left[\left(\lambda-\lambda_{o}\right)+\right.$ $\left.i \omega_{o}\right]$. Therefore, the effective frequency is entirely determined by the noise $\xi(t)$, which leads to a small effective frequency $\omega_{2}$, corresponding to the peak observed at $\omega_{2}=0$ in Fig. 5(c).

The density of points in the $\left(\omega_{1}, \omega_{2}\right)$ plane, shown in Fig. 5(d), demonstrates that in most cases $\omega_{2}<\omega_{1}$ (blue), with a small fraction of the cells having $\omega_{2}>\omega_{1}$ (black). We find a Pearson correlation coefficient between $\omega_{1}$ and $\omega_{2}$ of -0.28 ( $p$ value 0.17 ) in the experiment and 0.07 ( $p$ value 0 ) in numerical simulations. Taken together, this shows a qualitative resemblance between the measurements in Figs. 5(a) and 5(b) and simulations in Figs. 5(c) and 5(d). In particular, it justifies our assumption that actin oscillations are slowed down by changing $\lambda \rightarrow\left(\lambda-\lambda_{o}\right)$, close to a Hopf bifurcation. Finally, based on the comparison between experiments and simulations, our analysis suggests that (1) the coupling strength between actin polymerization and its negative regulators [ $x(t)$ and $y(t)$, respectively] does not change during the transient response and (2) the central frequency $\omega_{o}$ does not vary between cells. The variations in the detected frequencies $\omega_{1,2}$ can be attributed to phase noise, which we discuss in detail in the Appendix.

\section{CONCLUSIONS}

The cAMP-induced dynamic reorganization of the actin cytoskeleton in $D$. discoideum has been intensively investigated over the past decades [34-36]. Its temporal evolution shows a biphasic profile with a first pronounced maximum in the cortical F-actin concentration 5-10 s after stimulation, followed by a second, less pronounced, and broader peak of variable duration that may last up to several minutes $[19,20,37]$. Also upstream signaling components such as phosphatidylinositol $(3,4,5)$-trisphosphate (PIP3) and the GTP binding protein Rac exhibit a biphasic time profile
$[21,22]$ that has also been addressed by theoretical models $[24,25]$.

In this work, we extended the investigation of the cAMPinduced actin response, focusing on $D$. discoideum cells that showed self-sustained polymerization cycles prior to stimulation. After stimulation, the actin system transiently leaves the limit cycle and shifts to another dynamical state. This transient can be divided into two regimes (biphasic profile) that are characterized by the amplitude and phase transient times $\tau_{a}$ and $\tau_{\phi}$. Similar to the biphasic profiles reported in the previous literature, we found that the duration of the second regime varies strongly $\left(\left\langle\tau_{\phi}\right\rangle \pm \sigma_{\tau_{\phi}}=65 \pm 48.7 \mathrm{~s}\right)$. The detected lowest and highest values of $\tau_{\phi}$ were 16 and $188 \mathrm{~s}$ respectively, which corresponds to approximately 1 to 14 oscillation cycles of the prestimulation state. The dynamics during the second regime of the response is characterized by a lower amplitude and frequency and is related to the well known cAMP-induced cringing response of D. discoideum cells [38].

The actin cytoskeleton in $D$. discoideum involves 138 proteins [39]. Given this high degree of complexity, it is currently not possible to propose a detailed mechanistic model for the mathematical description of the actin cytoskeleton. We therefore address this problem from a reductionist viewpoint. Relying on our observation that the actin cytoskeleton in $D$. discoideum operates close to an oscillatory instability [4], we based our approach on the normal form of a supercritical Hopf bifurcation and proposed the noisy Stuart-Landau oscillator as a phenomenological model that successfully captures the main dynamical features of oscillatory actin dynamics in $D$. discoideum [6]. Here we extended this model to account for the experimentally observed cAMP-induced biphasic transient in oscillatory cells. As only a small fraction of cells show autonomous oscillations, only limited amounts of data are available for a comparison between model and experiment. Nevertheless, we find agreement in several distinct qualitative features summarized in Figs. 4 and 5. Our reductionist approach thus suggests that external cAMP stimulation reduces indirectly and transiently the value of the bifurcation parameter $\lambda \rightarrow\left(\lambda-\lambda_{o}\right)$, via the intracellular signaling machinery. Thus, for most oscillatory cells the transient corresponds to a regime, where periodic activity is disrupted or solely maintained by noise. Note that previous experiments on the cAMP-induced actin response did not specifically focus on oscillatory cells but relied on native $D$. discoideum populations that are dominated by a majority of nonoscillatory cells. For the nonoscillatory cells, the bifurcation parameter is negative, $\lambda<0$. In these cells, a further reduction of $\lambda$ in response to the cAMP stimulus will be difficult to detect, as no qualitative change in the dynamical state is associated with it. Note that previous theoretical studies of coupled oscillators with chemotaxis have shown nontrivial effects $[40,41]$. For example, it was demonstrated that oscillators with higher frequencies can lead the translocation of a cluster of cells. Our results of actin oscillators that change their dynamical state in the presence of cAMP indicate a real-world example, where such effects may become relevant.

Even though the detailed molecular mechanism of the biphasic response remains elusive, our results support the hypothesis that the cAMP-induced transients observed here 

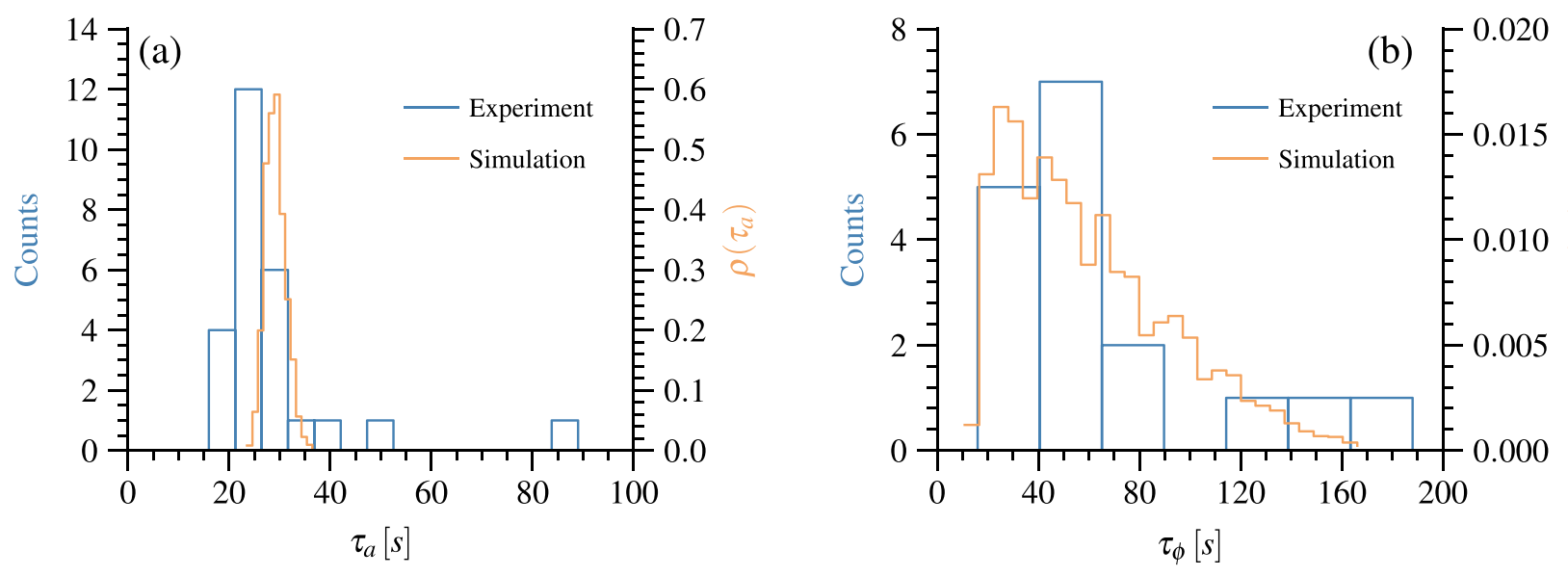

FIG. 6. Comparison of the experimentally and numerically derived amplitude and phase transient times. The experimental results are displayed as a histogram (blue) and the numerical results as a probability distribution function (orange) for $\tau_{a}$ (a) and $\tau_{\phi}$ (b).

primarily reflect the timescale of the signal transduction machinery. This is suggested by observations of intracellular signaling patterns in cells that have been treated with Latrunculin A to disrupt the actin cytoskeleton and exhibited timescales that were of the same order as the cAMP-induced transients observed here $[42,43]$. Similar timescales also emerged during gradient-induced Ras reorganization or mTORC2 activation $[44,45]$, suggesting that the transition to a lower amplitude
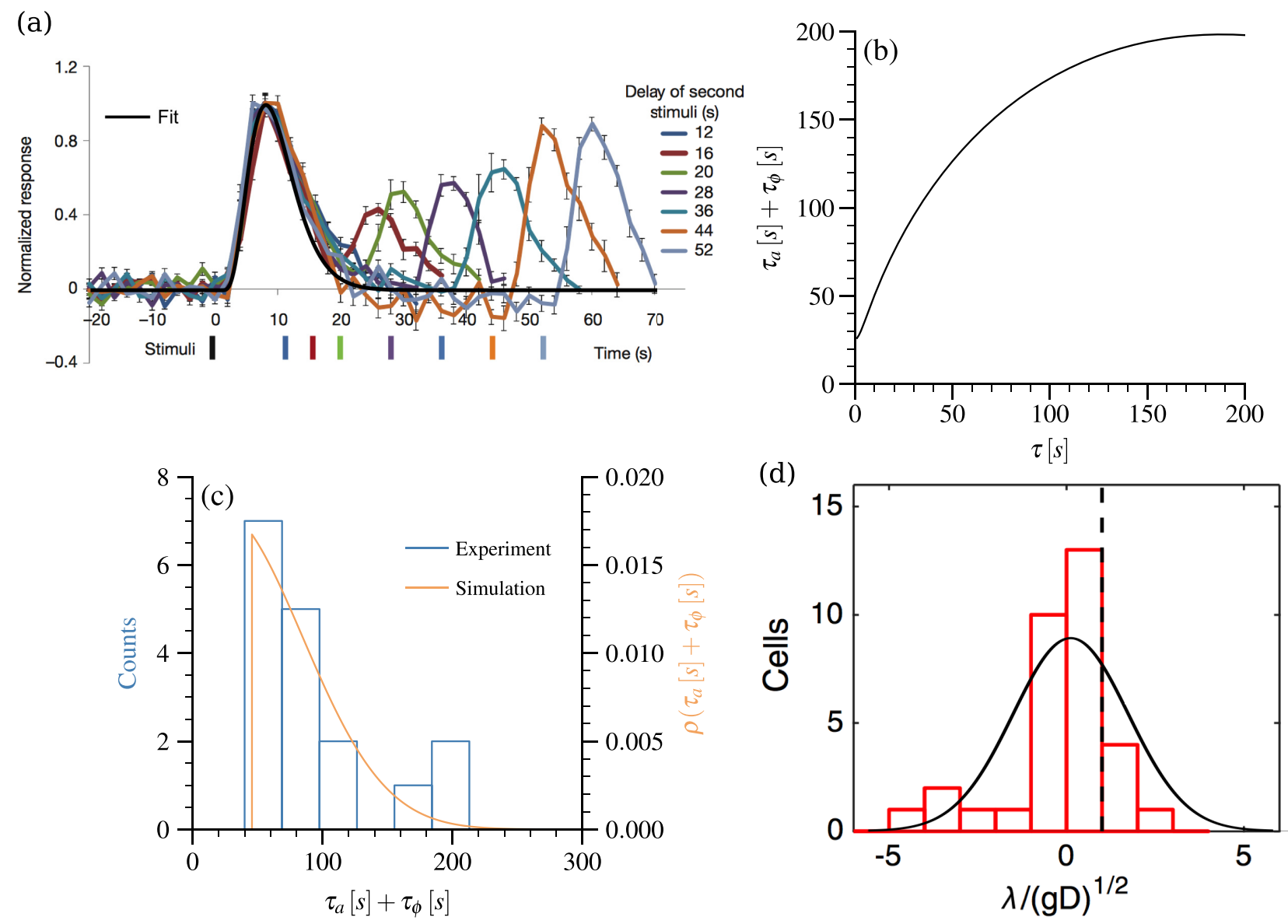

FIG. 7. Fitting procedure and derivation of parameters, based thereon. (a) Response of the chemotactic signaling system, measured by the translocation of the fluorescently labeled Ras binding domain (RBD-GFP) to two differently spaced pulses of cAMP. The signal input $s(t)$ was obtained by fitting (black line) the first responses. Figure reprinted and modified with permission from Ref. [33]. (b) Functional relation between the total transient time and the relaxation time $\tau$, given a fixed value of $K$. (c) Fit of the total transient time distribution resulting from $\rho(\tau)=\frac{\Theta\left(\tau-\tau_{1}\right)}{\tau_{2}} \exp ^{-\left(\tau-\tau_{1}\right) / \tau_{2}}$ and Eq. (A1). (d) Fit of a normal distribution with zero mean and a variance of 2.45 to the distribution of $\lambda / \sqrt{g D}$. Reprinted with permission from Ref. [6]. 
state may be related to the organization of the chemotactic machinery. Also, recently proposed models that rely on an oscillatory actin machinery guided by an upstream wavegenerating signaling systems support this view $[33,46]$.

\section{ACKNOWLEDGMENT}

We acknowledge funding for this work by the DFG SFB 937 "Collective behavior of soft and biological matter," Project No. A09.

\section{APPENDIX}

\section{Distributions of response timescales}

In Figs. 6(a) and 6(b), we show a comparison between the distributions of $\tau_{a}$ and $\tau_{\phi}$ obtained from experiments (blue bars) and from numerical simulations (orange lines). Our model roughly captures the positions of the dominant peaks in the experimental histograms. However, due to the limited number of data points in our experiments, we cannot conclusively compare the shapes of the distributions and base our comparison solely on the first two moments; see Sec. IV of the main text.

\section{Fitting procedure}

In this section, we describe the detailed procedure to constrain the parameters of Eqs. (5)-(8). The shape of $s(t)$, given by Eq. (6), was obtained by fitting the response of Ras to short-time stimuli of cAMP. The fitted data originated from an experiment described in Ref. [33]. Figure 7(a) shows the response of the fluorescently labeled Ras binding domain (RBD-GFP) to two consecutive 2-s pulses of cAMP. The fit of these data (black line) resulted in the parameters $s_{o}=1 / 4.69$ and $\tau_{s}=1.75$.

The distribution of relaxation time $\tau$ in Eq. (7) was assumed to decay exponentially, see Eq. (9), $\rho(\tau)=$ $\frac{\Theta\left(\tau-\tau_{1}\right)}{\tau_{2}} e^{-\left(\tau-\tau_{1}\right) / \tau_{2}}$. It is related to the distribution $\rho(T)$ of the total transient time $T$ by

$$
\rho(T)=\rho(\tau(T))\left|\frac{d \tau(T)}{d T}\right| .
$$

The dependence of the total transient time $T(\tau)$ on the relaxation time $\tau$, shown in Fig. 7(b), was obtained by fixing $K$ to a value of 0.035 ; see Eq. (8). The fitting parameters $\tau_{1}=7.8 \mathrm{~s}$ and $\tau_{2}=20.75 \mathrm{~s}$ in the distribution $\rho(\tau)$ are chosen such that the distribution $\rho(T)$ resulting from Eq. (A1) fits the experimentally obtained distribution of the total transient time $T=\tau_{a}+\tau_{\phi}$, which is shown in Fig. 7(c).

For completeness, we recall that the distribution of the dimensionless parameter $\Lambda=\lambda / \sqrt{g D}$ has been simplified here as a normal distribution; see Eq. (10).

The oscillator frequency $\omega_{o}$ of Eq. (5) is set to the average observed frequency $\langle\omega\rangle=\omega_{o}$. The parameter $\lambda_{o}$ [Eq. (8)] was estimated by plotting $\mu_{a}^{(1)}$ against $\mu_{a}^{(2)}$, see Fig. $8(\mathrm{a})$, and comparing it with the theoretically derived curves [Figs. 8(b)$8(\mathrm{e})]$. The transition from a steep to a smaller slope, as $\mu_{a}^{(1)}$ is increased, is most pronounced in Fig. 8(e) with $\lambda_{o}=6 \sqrt{g D}$. Therefore, we chose the value $\lambda_{o}=6 \sqrt{g D}$ as a good approximation. Finally, the remaining free parameter in Eq. (5) was set to $\tau_{r}=1 / 2 \mathrm{~s}$.

\section{Phase slips}

The rotation number in the numerical simulations, defined as the number of oscillator cycles, depends on the sampling
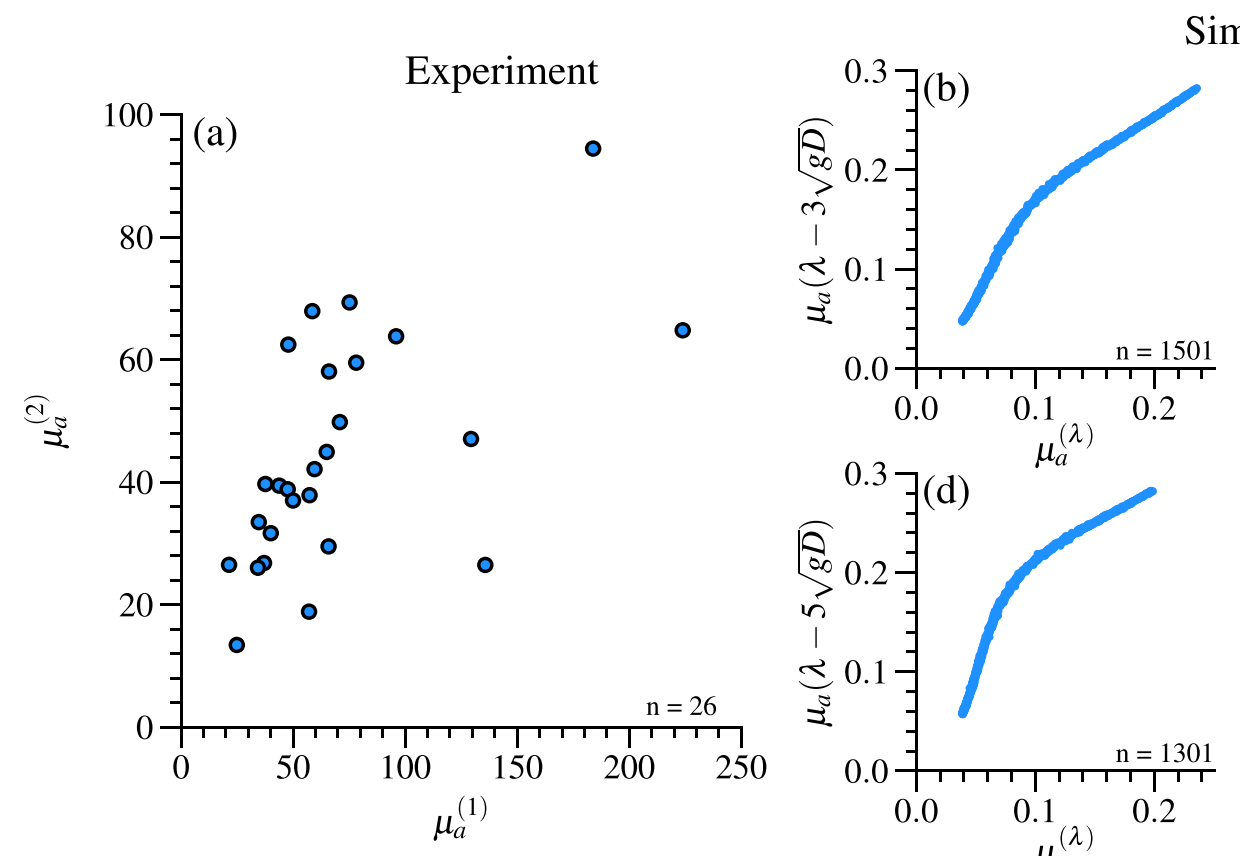

Simulation
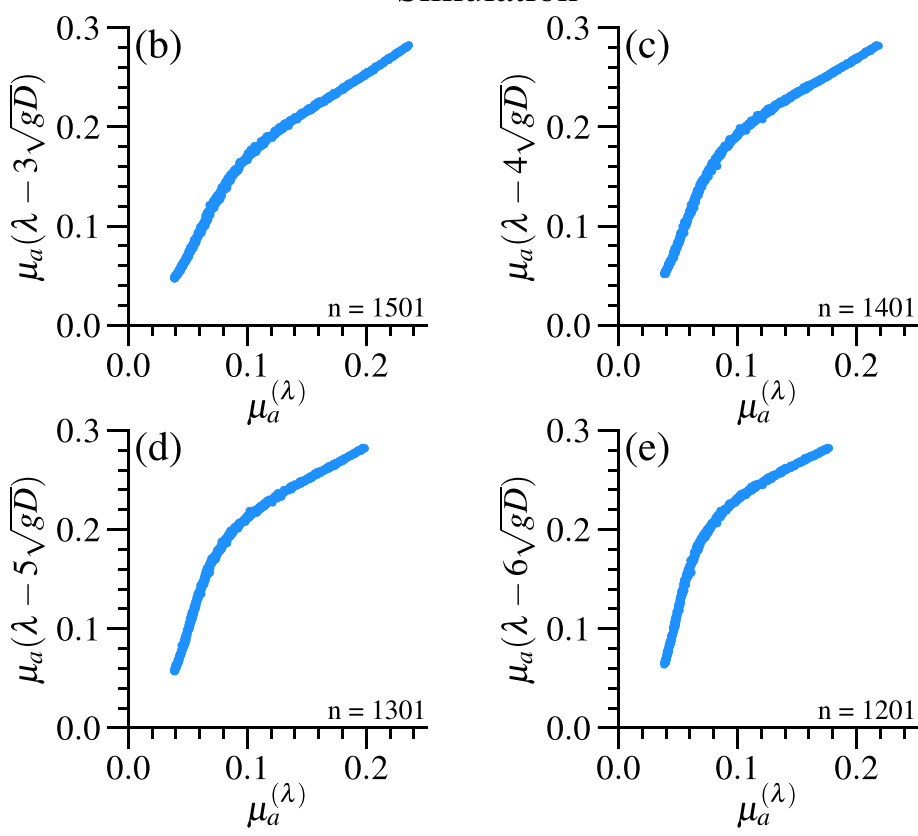

FIG. 8. Comparison of experimentally and numerically derived amplitude means. (a) Relation of the measured amplitude means before stimulation $\left(\mu_{a}^{(1)}\right)$ and during $\tau_{\phi}\left(\mu_{a}^{(2)}\right)$. [(b)-(e)] Amplitude means obtained by evaluating Eq. (3) with the following parameter set: (b) $\lambda_{o}=$ $3 \sqrt{g D}$, (c) $\lambda_{o}=4 \sqrt{g D}$, (d) $\lambda_{o}=5 \sqrt{g D}$, and (e) $\lambda_{o}=6 \sqrt{g D}$. 
Fine sampling
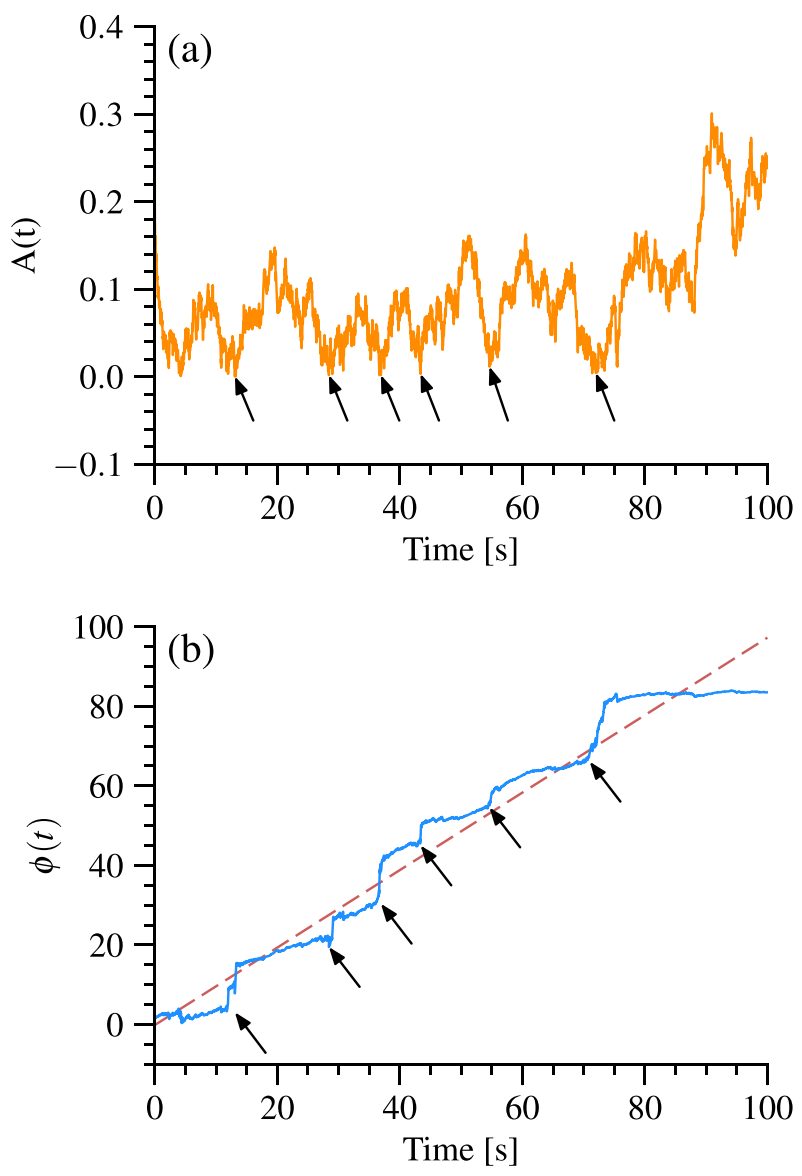

Coarse sampling
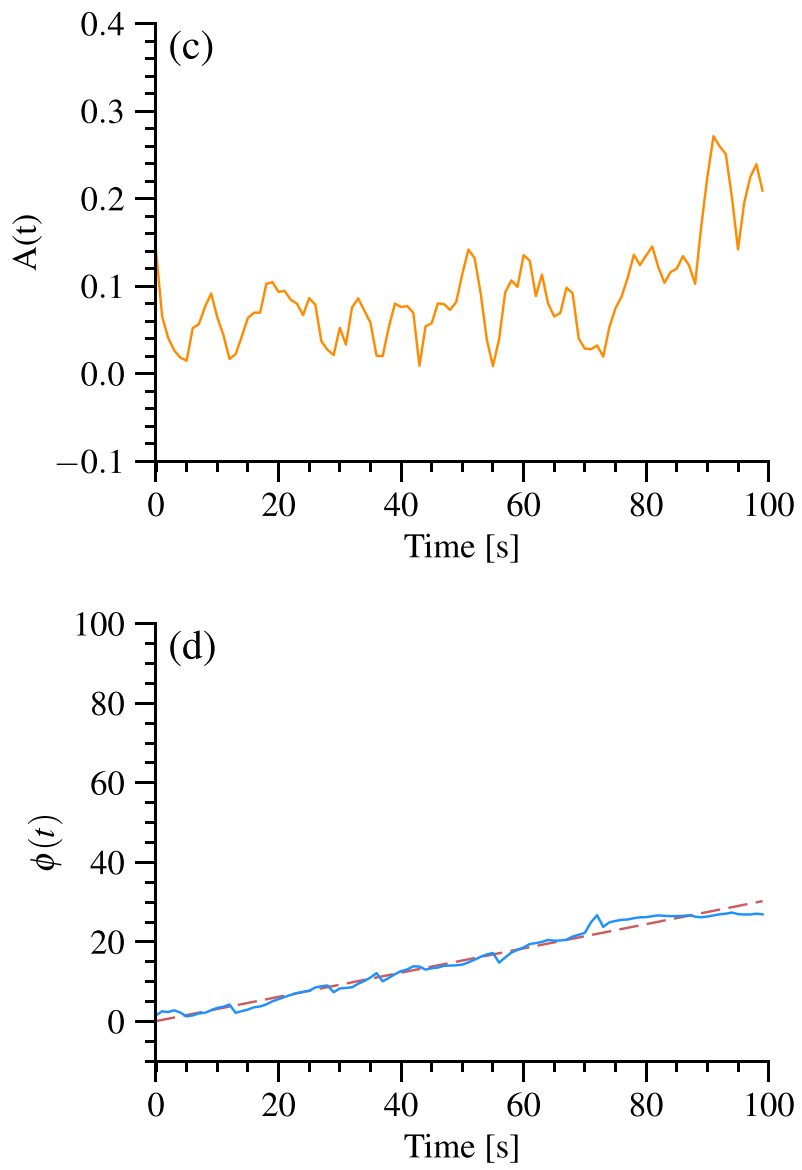

FIG. 9. Comparison of fine and coarse sampling. Amplitude $A(t)$ (a) and phase $\phi(t)$ (b) resulting from a numerical simulation with fine sampling $(\Delta t=0.01 \mathrm{~s})$. Black arrows mark phase slips. [(c), (d)] Amplitude $A(t)$ and phase $\phi(t)$ obtained from a numerical simulation with coarse sampling $(\Delta t=1.0 \mathrm{~s})$. Red dashed lines in panels (b) and (d) indicate a linear fit to the phase $\phi(t)$.

acquisition rate. The amplitude $A(t)$ and phase $\phi(t)$, plotted in Figs. 9(a) and 9(b), were calculated with a high sampling rate $(\Delta t=0.01 \mathrm{~s})$. The phase shows several phase slips (i.e., discontinuities), which are marked by black arrows in Fig. 9(b). These result from amplitude fluctuations close to
$A(t)=0$. The detected number of oscillator cycles in this case yields $80 / 2 \pi=12.73$.

On the other hand, the number of values close to $A(t)=0$ is reduced if the numerically derived time series are downsampled to the same rate as in experiment $(\Delta t=1.0 \mathrm{~s})$. The
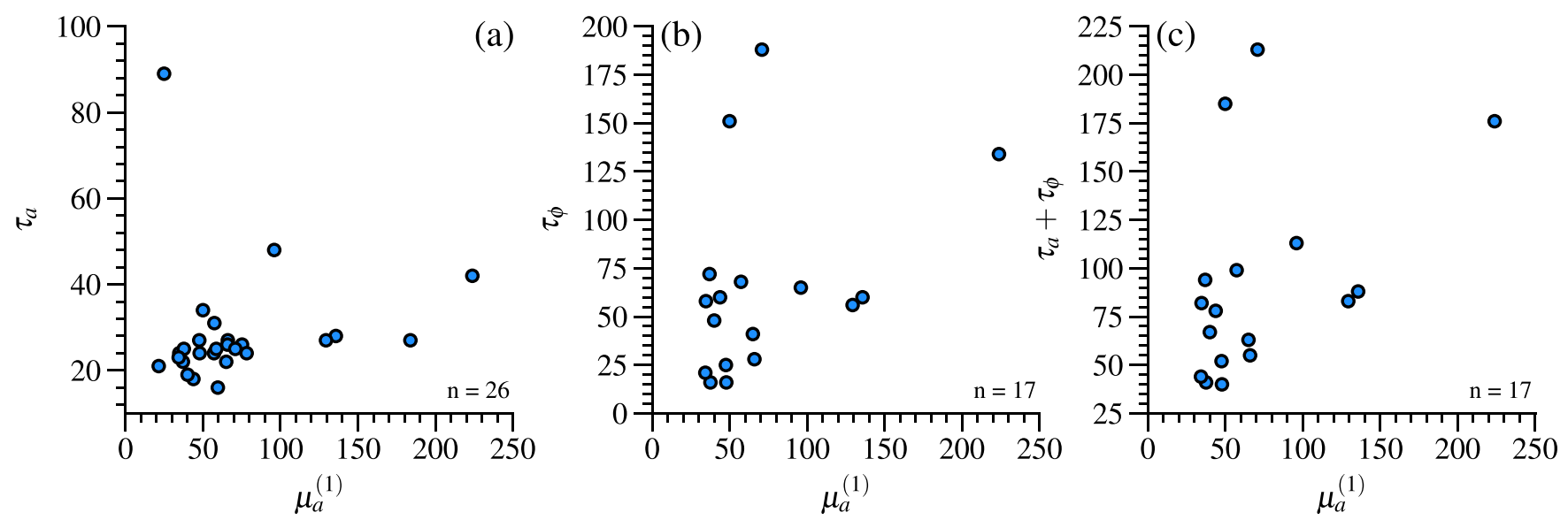

FIG. 10. Dependence of the mean fluorescence intensity $\left(\mu_{a}^{(1)}\right)$ prior to stimulation on the different response timescales. (a) Comparison of $\mu_{a}^{(1)}$ to the amplitude transient time $\tau_{a}$, (b) to the phase transient time $\tau_{\phi}$, and (c) to the sum of both transient times. 

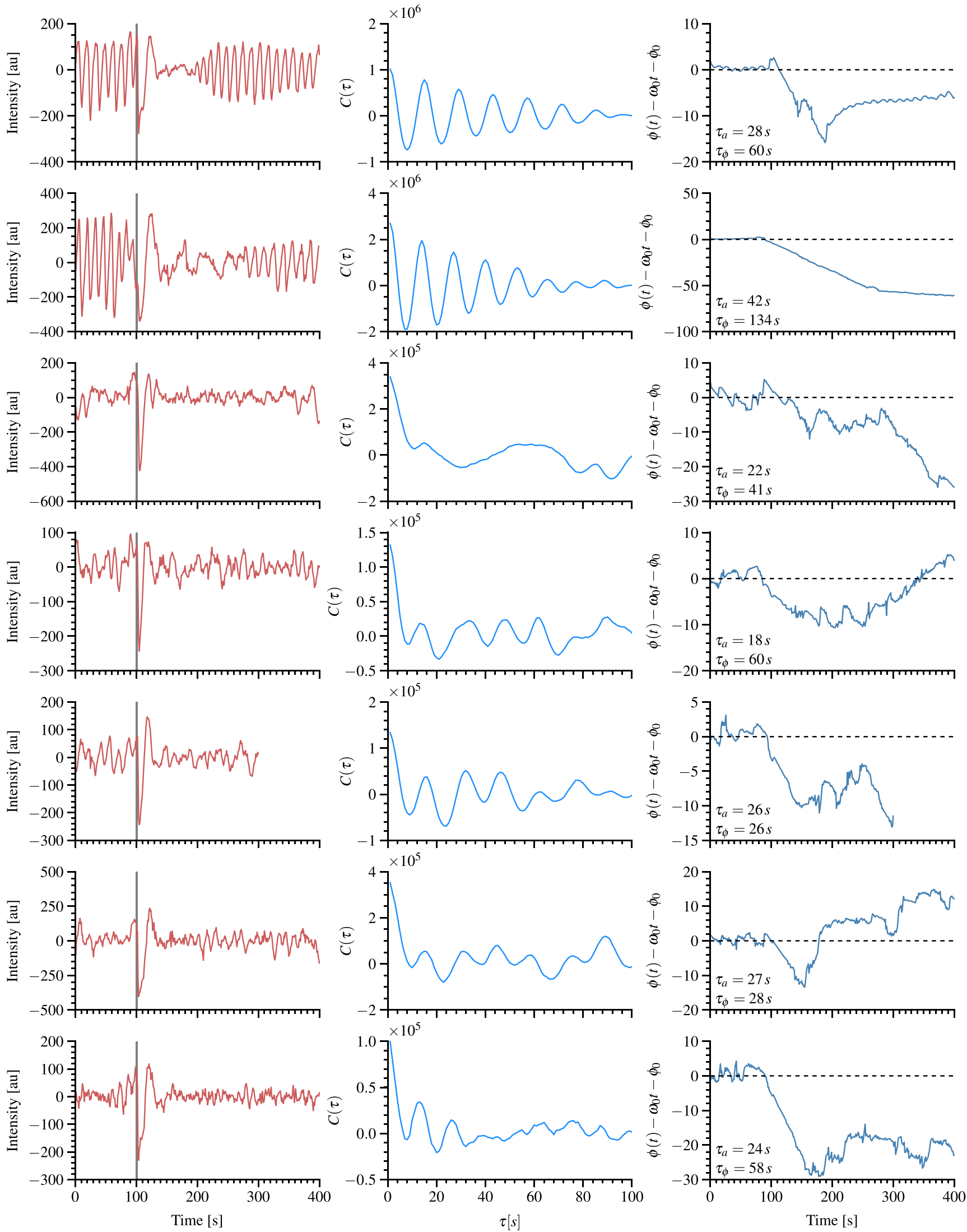

FIG. 11. Individual fluorescent time traces used for data analysis. Left column shows the individual time signals after detrending and the middle column shows the corresponding autocorrelation functions $[C(\tau)]$, calculated over the first $100 \mathrm{~s}$ of the time signal. The right column gives the evolution of the phase. All cases shown in this figure are decelerating the phase. The time of stimulation $(t=100 \mathrm{~s})$ is marked by a gray bar in the time signals. 

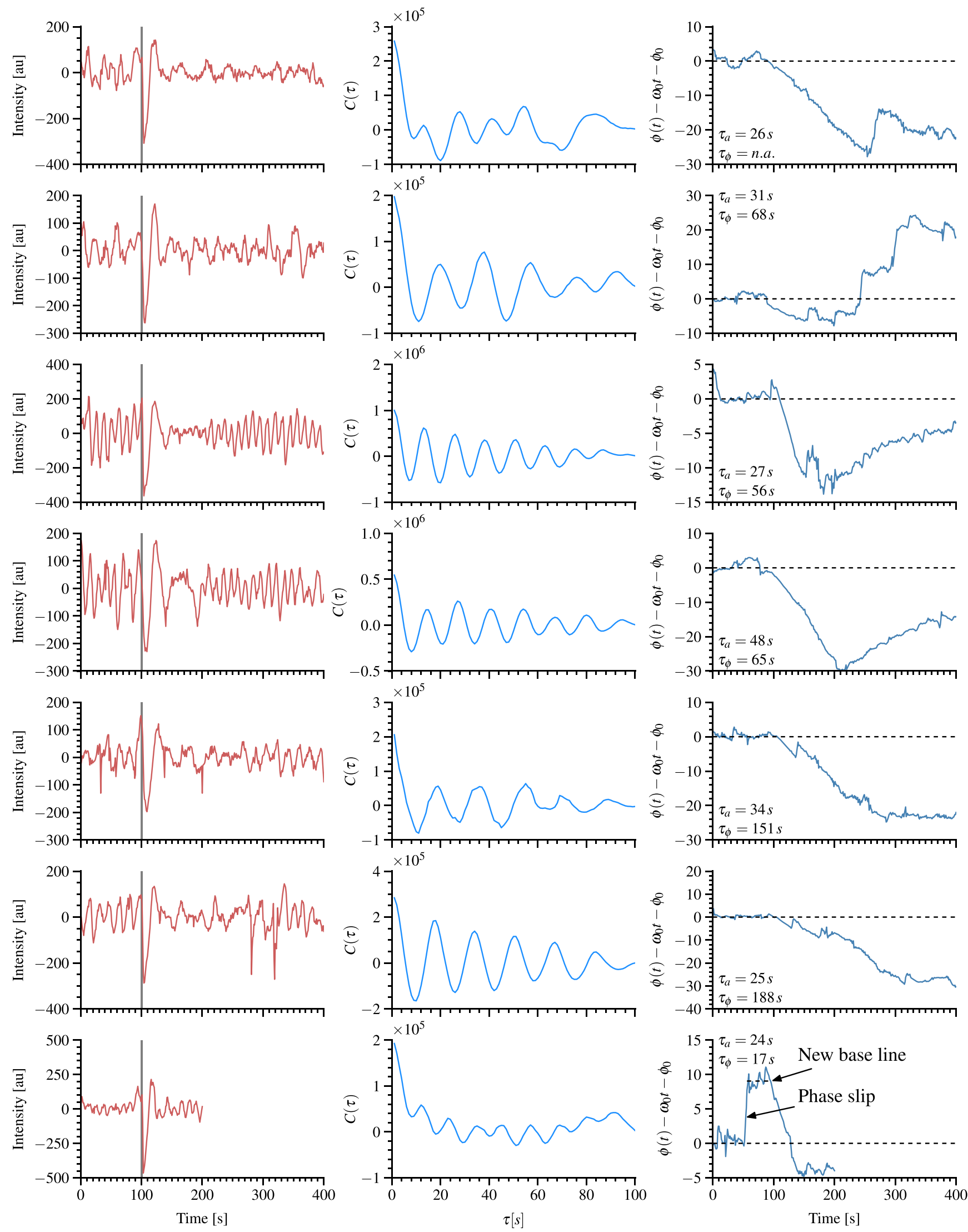

FIG. 12. Individual fluorescent time traces used for data analysis. (Continued from Fig. 11.) 

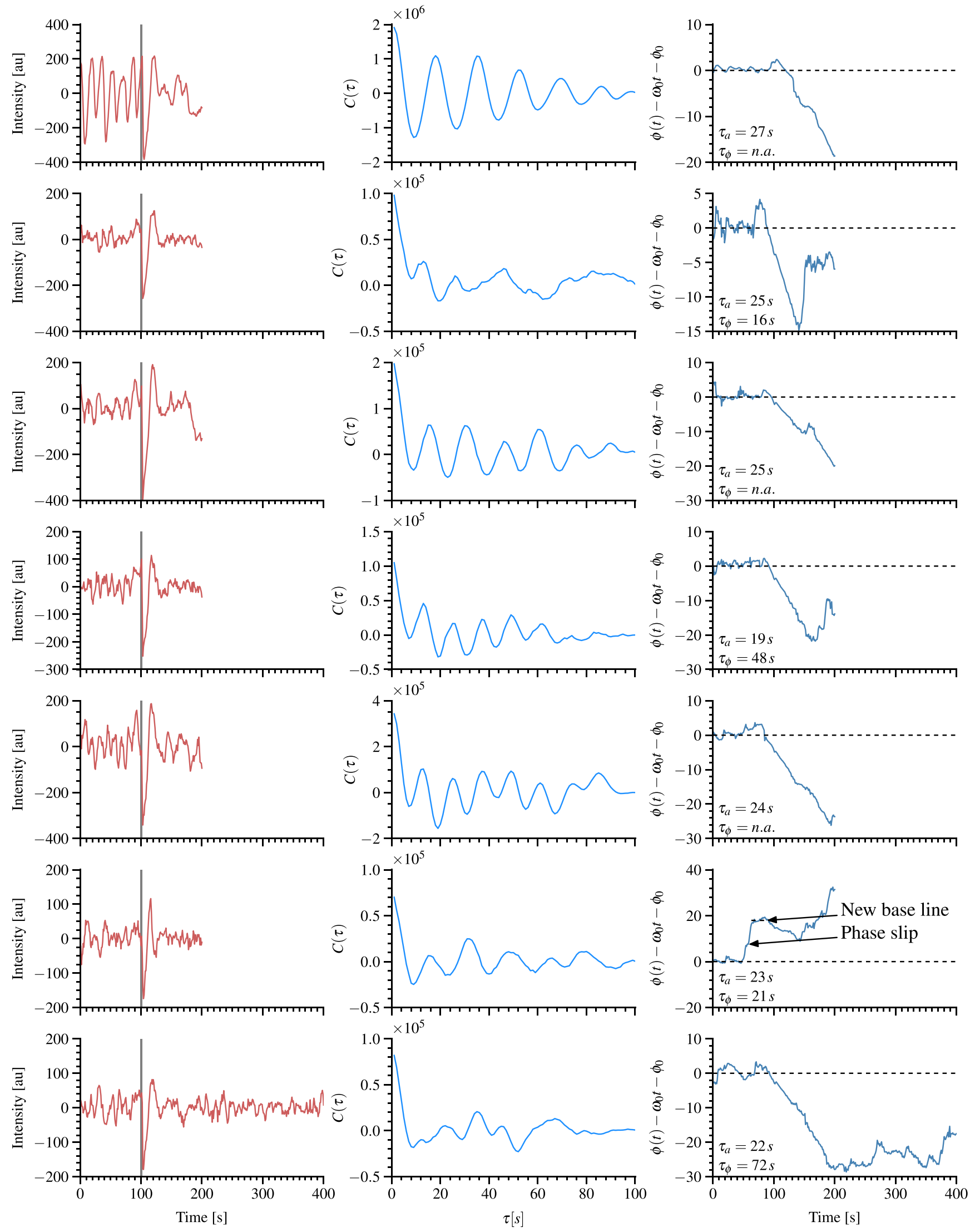

FIG. 13. Individual fluorescent time traces used for data analysis. (Continued from Fig. 11.) 

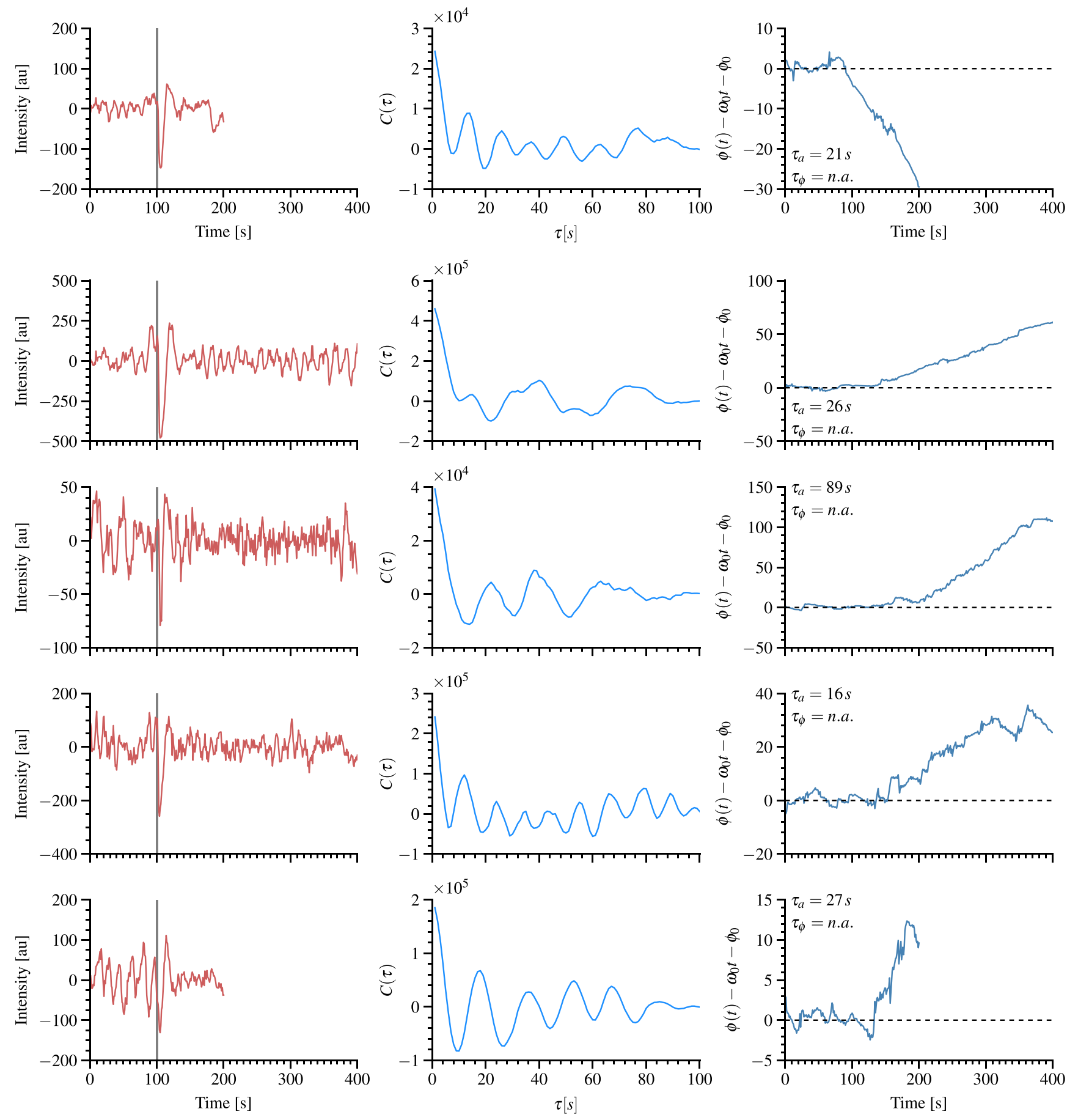

FIG. 14. Individual fluorescent time traces used for data analysis. (Continued from Fig. 11.) The lower four rows show the cases of accelerating phase after stimulation.

number of phase slips diminishes dramatically in this case and as a consequence the number of oscillator cycles is reduced to $30 / 2 \pi=2.77$. Therefore, the detected frequency $\omega$, obtained by fitting a straight line to the phase $\phi(t)$ is lower for the coarse sampled signal [compare Figs. 9(c) and 9(d), where the red dashed line indicates the linear fit]. Furthermore, the fit accuracy is increased by the coarse sampling. Therefore, we used the experimental sampling rate to analyze the detected frequency distribution in both the experimentally and the numerically obtained time series.

\section{Correlation of amplitude mean and transient timescales}

The dependence of the mean fluorescence intensity $\left(\mu_{a}^{(1)}\right)$ prior to stimulation on the different response timescales $\left(\tau_{a}\right.$ and $\tau_{\phi}$ ) are shown in Fig. 10. No significant correlations were found.

\section{Time series}

All experimental time series, which were identified as oscillatory before stimulation, along with their respective 


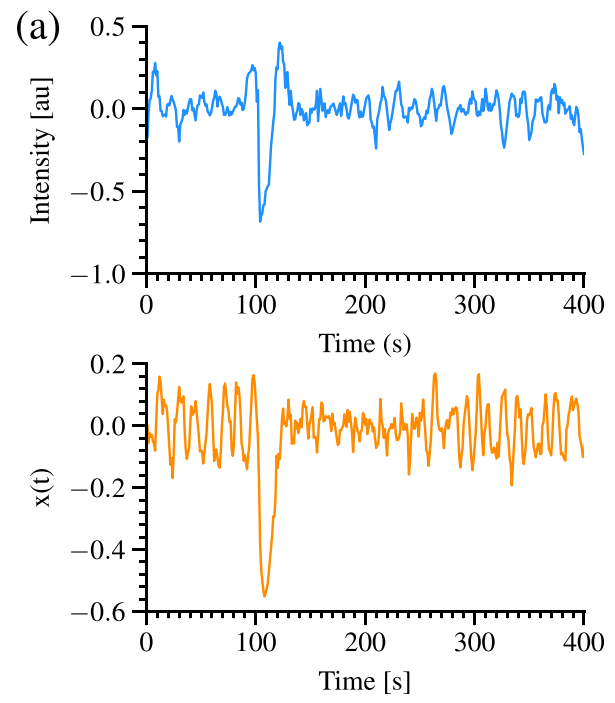

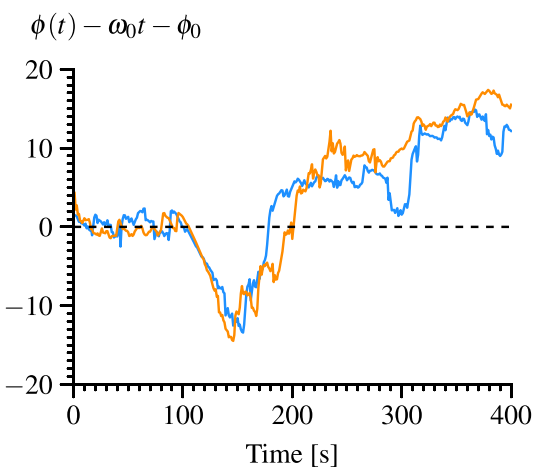

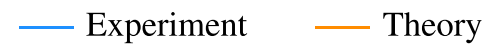

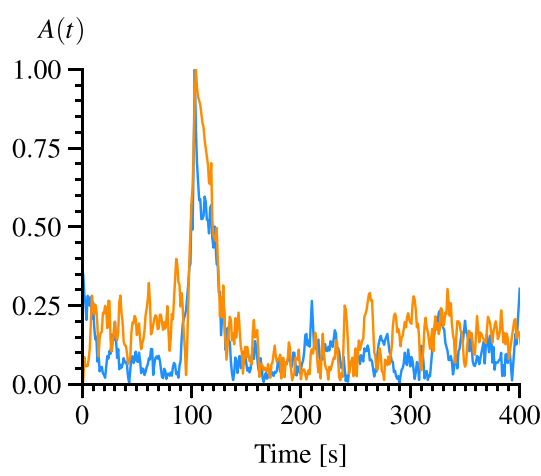
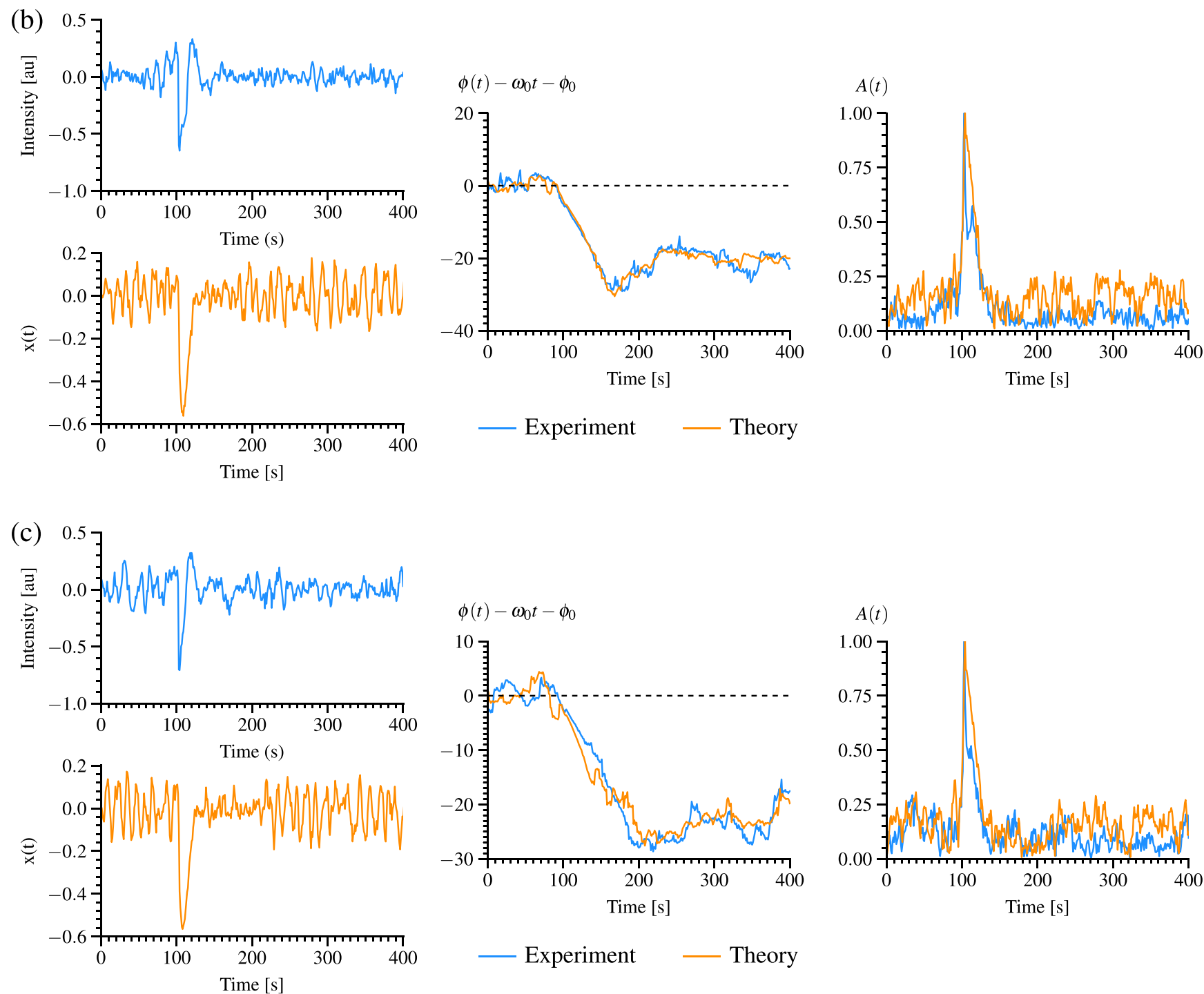

Experiment Theory

FIG. 15. Comparison of experimental and numerical results, selected according to minimization of the error function (A2) as explained in the text. In panels (a), (b), and (c), three examples are displayed. On the left, blue and orange curves display the experimental and numerical time traces, respectively. In the middle and on the right, phase and amplitude drifts are shown, respectively, using the same color coding. 
autocorrelation functions $[A C(t)]$ are shown in Figs. 11-14. The $A C(t)$ are given only for the initial $100 \mathrm{~s}$ of the respective time series.

\section{Comparison between simulations and experimental data}

We have simulated 4684 realizations of the model given by Eqs. (5)-(8). To check if the model reproduced the main features of the experimental data, we defined the following error function,

$$
E^{(i, j)}=\frac{\left|A_{\exp }^{(i)}-A_{\text {sim }}^{(j)}\right|}{\left|A_{\exp }^{(i)}\right|} \frac{\left|\psi_{\exp }^{(i)}-\psi_{\text {sim }}^{(j)}\right|}{\left|\psi_{\exp }^{(i)}\right|},
$$

where $A_{\exp }^{(i)}$ and $\psi_{\exp }^{(i)}$ correspond to the experimental time traces of the amplitude and phase drifts, respectively, and the index $i$ corresponds to the experiment number $(1 \leqslant i \leqslant 22)$. The amplitude $A_{\exp }^{(i)}$ was normalized by dividing it with its maximum value. At the same time, $A_{\text {sim }}^{(j)}$ and $\psi_{\text {sim }}^{(j)}$ correspond to the simulated time traces of the amplitude and phase drifts, respectively, and the index $j$ corresponds to the simulation number $(1 \leqslant j \leqslant 4684)$. We applied the same criteria to the simulated realizations concerning the decay of the autocorrelation function and applied the same sampling rate (see main text for details).

We have assigned to each experimental time trace a numerical time trace by finding $\min \left[E^{(i, j)}\right]$. In Fig. 15, we show three examples of experimental time traces along with the numerical simulations that were chosen in this way. The numerical time traces reproduce also details of the experimental traces. In particular, we note that in a small set of numerical simulations, the frequency increased after stimulation, i.e., $\omega_{2}>\omega_{1}$, similar to the experimental results. This demonstrates that both cases observed in experiment, $\omega_{2}<\omega_{1}$ and $\omega_{2}>\omega_{1}$, emerge as part of the same oscillatory mechanism.
[1] P. N. Devreotes, S. Bhattacharya, M. Edwards, P. A. Iglesias, T. Lampert, and Y. Miao, Annu. Rev. Cell Dev. Biol. 33, 103 (2017).

[2] J. M. Nichols, D. Veltman, and R. R. Kay, Curr. Opin. Cell Biol. 36, 7 (2015).

[3] H. F. Hsu, A. Krekhov, M. Tarantola, C. Beta, and E. Bodenschatz, New J. Phys. 21, 113055 (2019).

[4] C. Westendorf, J. Negrete, A. J. Bae, R. Sandmann, E. Bodenschatz, and C. Beta, Proc. Nat. Acad. Sci. USA 110, 3853 (2013).

[5] H. F. Hsu, E. Bodenschatz, C. Westendorf, A. Gholami, A. Pumir, M. Tarantola, and C. Beta, Phys. Rev. Lett. 119, 148101 (2017)

[6] J. Negrete, Jr., A. Pumir, H.-F. Hsu, C. Westendorf, M. Tarantola, C. Beta, and E. Bodenschatz, Phys. Rev. Lett. 117, 148102 (2016).

[7] T. Mora and W. Bialek, J. Stat. Phys. 144, 268 (2011).

[8] J. Soriano, C. Colombo, and A. Ott, Phys. Rev. Lett. 97, 258102 (2006).

[9] D. Krotov, J. O. Dubuis, T. Gregor, and W. Bialek, Proc. Natl. Acad. Sci. USA 111, 3683 (2014).

[10] C. Beta and K. Kruse, Annu. Rev. Condens. Matter Phys. 8, 239 (2017).

[11] Y. E. Antebi, N. Nandagopal, and M. B. Elowitz, Curr. Opin. Syst. Biol. 1, 16 (2017).

[12] L. Bosgraaf and P. J. M. Van Haastert, PLoS ONE 4, e5253 (2009).

[13] L. Bosgraaf and P. J. M. Van Haastert, PLoS ONE 4, e6842 (2009).

[14] C. Beta, D. Wyatt, W. J. Rappel, and E. Bodenschatz, Anal. Chem. 79, 3940 (2007).

[15] C. Beta and E. Bodenschatz, Eur. J. Cell Biol. 90, 811 (2011).

[16] J. Prassler, A. Murr, S. Stocker, J. Faix, J. Murphy, and G. Marriott, Mol. Biol. Cell 9, 545 (1998).

[17] N. Schneider, I. Weber, J. Faix, J. Prassler, A. MüllerTaubenberger, J. Köhler, E. Burghardt, G. Gerisch, and G. Marriott, Cell Motil. 56, 130 (2003).
[18] M. Etzrodt, H. Ishikawa, J. Dalous, M. Annette, T. Bretschneider, and G. Gerisch, Febs Lett. 580, 6707 (2006).

[19] A. Hall, A. Schlein, and J. Condeelis, J. Cell. Biochem. 37, 285 (1988).

[20] L. Chen, C. Janetopoulos, Y. Huang, M. Iijima, J. Borleis, and P. Devreotes, Mol. Biol. Cell 14, 5028 (2003).

[21] K. C. Park, F. Rivero, R. Meili, S. Lee, F. Apone, and R. A. Firtel, EMBO J. 23, 4177 (2004).

[22] M. Postma, J. Roelofs, J. Goedhart, T. W. J. Gadella, A. J. W. G. Visser, and P. J. M. Van Haastert, Mol. Biol. Cell 14, 5019 (2003).

[23] M. Postma, J. Roelofs, J. Goedhart, H. M. Loovers, A. J. W. G. Visser, and P. J. M. Van Haastert, J. Cell Sci. 117, 2925 (2004).

[24] Y. Xiong, C. H. Huang, P. A. Iglesias, and P. N. Devreotes, Proc. Natl. Acad. Sci. USA 107, 17079 (2010).

[25] I. Hecht, D. A. Kessler, and H. Levine, Phys. Rev. Lett. 104, 158301 (2010).

[26] J. Guckenheimer and P. Holmes, Nonlinear Oscillations, Dynamical Systems, and Bifurcations of Vector Fields (Springer, New York, 2002).

[27] S. Strogatz, Nonlinear Dynamics and Chaos: With Applications to Physics, Biology, Chemistry and Engineering (Westview Press, Boulder, 2014).

[28] S. Diez, G. Gerisch, K. Anderson, M. Annette, and T. Bretschneider, Proc. Nat. Acad. Sci. USA 102, 7601 (2005).

[29] G. Gerisch, R. Albrecht, C. Heizer, S. Hodgkinson, and M. Maniak, Curr. Biol. 5, 1280 (1995).

[30] A. Konzok, I. Weber, E. Simmeth, U. Hacker, M. Maniak, and M. Annette, J. Cell. Biol. 146, 453 (1999).

[31] A. Bae, C. Beta, and E. Bodenschatz, Lab Chip 9, 3059 (2009).

[32] A. Levchenko and P. A. Iglesias, Biophys. J. 82, 50 (2002).

[33] C. H. Huang, M. Tang, C. Shi, P. A. Iglesias, and P. N. Devreotes, Nat. Cell Biol. 15, 1307 (2013).

[34] S. J. McRobbie and P. C. Newell, Biochem. Biophys. Res. Commun. 115, 351 (1983). 
[35] S. J. McRobbie and P. C. Newell, J. Cell Sci. 68, 139 (1984).

[36] S. Yumura, Cell Struct. Funct. 19, 143 (1994).

[37] J. Condeelis, A. Hall, A. Bresnick, V. Warren, R. Hock, H. Bennett, and S. Ogihara, Cell Motility 10, 77 (1988).

[38] K. F. Swaney, C.-H. Huang, and P. N. Devreotes, Annu. Rev. Biophys. 39, 265 (2010).

[39] L. Eichinger, J. A. Pachebat, G. Glöckner, M.-A. Rajandream, R. Sucgang, M. Berriman, J. Song, R. Olsen, K. Szafranski, Q. $\mathrm{Xu}$, B. Tunggal, S. Kummerfeld, M. Madera, B. A. Konfortov, F. Rivero, A. T. Bankier, R. Lehmann, N. Hamlin, R. Davies, P. Gaudet et al., Nature (London) 435, 43 (2005).
[40] S. Sawai and Y. Aizawa, J. Phys. Soc. Jpn. 67, 2557 (1998).

[41] D. Tanaka, Phys. Rev. Lett. 99, 134103 (2007).

[42] G. Gerisch, T. Bretschneider, A. Müller-Taubenberger, E. Simmeth, M. Ecke, S. Diez, and K. Anderson, Biophys. J. 87, 3493 (2004).

[43] Y. Arai, T. Shibata, S. Matsuoka, M. J. Sato, T. Yanagida, and M. Ueda, Proc. Nat. Acad. Sci. USA 107, 12399 (2010).

[44] A. Kortholt, I. Keizer-Gunnink, R. Kataria, and P. J. M. V. Haastert, J. Cell Sci. 126, 4502 (2013).

[45] H. Senoo, Y. Kamimura, R. Kimura, A. Nakajima, S. Sawai, H. Sesaki, and M. Iijima, Nat. Cell Biol. 21, 867 (2019).

[46] M. Ecke and G. Gerisch, Small GTPases 10, 72 (2019). 